\title{
Thermodynamics of Gas Turbine Cycles With Analytic Derivatives in OpenMDAO
}

\author{
Justin Gray, ${ }^{*}$ Jeffrey Chin,${ }^{\dagger}$ Tristan Hearn,${ }^{\ddagger}$ Eric Hendricks,${ }^{\S}$ Thomas Lavelle, $₫$ \\ NASA Glenn Research Center, Cleveland, $\mathrm{OH}$ \\ Joaquim R. R. A. Martins" \\ University of Michigan, Ann Arbor, MI
}

\begin{abstract}
A new equilibrium thermodynamics analysis tool was built based on the CEA method using the OpenMDAO framework. The tool provides forward and adjoint analytic derivatives for use with gradient based optimization algorithms. The new tool was validated against the original CEA code to ensure an accurate analysis and the analytic derivatives were validated against finite-difference approximations. Performance comparisons between analytic and finite difference methods showed a significant speed advantage for the analytic methods. To further test the new analysis tool, sample optimizations were performed performed to find the optimal air-fuel equivalence ratio, $\phi$, maximizing combustion temperature for a range of different pressures. Collectively, the results demonstrate the viability of the new tool to serve as the thermodynamic backbone for future work on a full propulsion modeling tool well suited for use with gradient based optimization.
\end{abstract}

\section{Nomenclature}

() $\quad$ quantity for the $i^{\text {th }}$ element

() $)_{j} \quad$ quantity for the $j^{\text {th }}$ chemical species

()$_{k} \quad$ alternate subscript for the $k^{t h}$, chemical species

$\gamma \quad$ ratio of specific heats $\left(C_{p} / C_{v}\right)$

$\lambda \quad$ lagrange multipliers

$\mathcal{R}$ residual function

$\mu \quad$ chemical potential energy

$\phi \quad$ air-fuel equivalence Ratio

$\pi \quad$ modified Lagrange multipliers

$\rho$ density

$a_{i j} \quad$ stoichiometric constant for the $i^{\text {th }}$ element in the $j^{\text {th }}$ species

$b \quad$ moles of an element summed across all species in a gas

$b^{\circ} \quad$ moles of an element summed across all species in a gas at the initial composition

$C_{p} \quad$ specific heat at constant pressure

$C_{v} \quad$ specific heat at constant volume

$G \quad$ Lagrangian of the Gibbs free energy minimization

$g \quad$ Gibbs free energy

*Aerospace Engineer Propulsion Systems Analysis Branch, Doctoral Pre-Candidate, Department of Aerospace Engineering, University of Michigan, AIAA Member

${ }^{\dagger}$ Aerospace Engineer PSA Branch, AIAA Member

¥Aerospace Engineer PSA Branch

$\S$ Aerospace Engineer, PSA Branch

I Aerospace Engineer PSA Branch

\| Professor, Department of Aerospace Engineering, AIAA Associate Fellow 
$h \quad$ specific enthalpy

$H^{\circ} \quad$ enthalpy of a species as a function of $T$

$h_{o} \quad$ input specified enthalpy

$n$ concentration of chemical species ( $\mathrm{kg}$-mol $/ \mathrm{kg}$-mixture)

$N_{e} \quad$ total number of elements

$N_{s} \quad$ total number of chemical species

$P \quad$ Pressure

$P_{a} \quad$ Pressure at standard conditions (1.01325 Bar)

$R \quad$ universal gas constant

$S^{\circ} \quad$ entropy of a species as a function of $T$

$S_{o} \quad$ input specified entropy

$T \quad$ Temperature

$U \quad$ vector of state variables in the chemical equilibrium solve

\section{Introduction}

A new generation of aircraft concepts have been proposed, exploiting propulsion airframe integration for higher efficiency. These concepts, such as over wing nacelles $\sqrt{12}$ and boundary layer ingestion, $\sqrt[3]{1}$ couple the thermodynamic performance of the propulsion system with the aerodynamic performance of the airframe. This, in turn, necessitates a coupling of the aerodynamic and propulsion analysis tools for the aircraft design and motivates the application of Multidisciplinary Design Analysis and Optimization (MDAO) to navigate the potentially large design space.

In order to apply MDAO, it is necessary to have a set of analysis tools well suited to optimization. This generally means that tools needs to be numerically stable, be capable of giving physically meaningful results even when given somewhat poor designs, and be reasonably computationally efficient. In cases where gradient based optimization will be employed, it is also important that analysis tools have smooth and differentiable formulations and it is highly beneficial if they provides analytic derivatives as well.

The aerodynamics community has developed a set of Computational Fluid Dynamics (CFD) tools, such as Fun3d, ${ }^{4}$ SU2, 5 and SumAD, ${ }^{[6}$ with adjoint analytic derivatives tailored for gradient based optimization. But the state-of-the-art propulsion analysis tool, the Numerical Propulsion System Simulation (NPSS) 7 is not as well developed for optimization applications. NPSS is a flexible tool for modeling the thermodynamic cycles of propulsions systems and predicting their overall performance throughout a range of flight conditions. While it has been used in a number of integrated propulsion airframe studies, the methods used have been limited to lower fidelity aerodynamic models, loose coupling, and mostly gradient free optimizers. Felder et. al. applied NPSS to boundary layer ingestion and distributed propulsion concepts, though this did not directly couple the propulsion and airframe models. ${ }^{3}$ Allison et. al. have done extensive work integrating NPSS into the conceptual design for military aircraft, but have focused more on design space exploration than optimization $\sqrt[8]{9}$ Geiselhart et. al. used NPSS as part of a low-boom supersonic transport design optimization, but noted numerical stability as a primary motivation for using gradient free optimization methods $\frac{10}{0}$

One of the major challenges with integrating NPSS into the gradient based optimization methods used for aerodynamic shape optimization is that it relies on finite-difference approximations to compute derivatives. The finite-difference method has two primary draw-backs: its computational cost grows linearly with the size of the design space and it can have numerical accuracy issues. Hendricks et. al. highlighted acute inaccuracy of finite difference gradients when optimizing a four stage power turbine, using meanline turbomachinery models built in NPSS 11 To address the challenges of applying NPSS in MDAO applications, a new cycle analysis tool, named PyCycle, is being developed with the goal of maintaining the thermodynamic cycle analysis capabilities of NPSS but also providing analytic derivatives for MDAO applications.

PyCycle is being built with the OpenMDAO framework, ${ }^{12}$ leveraging its built in solvers, data passing, and automatic multidisciplinary derivatives capability. Like NPSS, Pycycle will be composed of a set of propulsion elements (inlet, compressor, combustor, turbine, etc.) which can be linked together in a variety of ways to model a full propulsion system. Further examination of the underlying physics of an element reveals a finer structure. For example, calculations in the compressor element can be broken down into five steps:

1. determine the exit pressure based on the input pressure ratio 
2. solve for ideal exit thermodynamic conditions using the exit pressure and assuming constant entropy

3. compute real exit enthalpy given efficiency

4. solve for real exit thermodynamic conditions given exit enthalpy and pressure

5. compute the shaft power required given the change in enthalpy across compressor

Steps 2 and 4 are thermodynamic solves and steps 1, 3 and 5 are engineering relationships. The thermodynamics solves compute properties of the working fluid (enthalpy, entropy, temperature, pressure, etc.) given any two thermodynamics states. All propulsion elements can be broken down into some set of thermodynamic solves separated by engineering calculations, although the specific combinations vary from element to element. This makes the thermodynamic solves a fundamental underlying requirement for any propulsion analysis code. This, before work can begin on the propulsion elements for PyCycle the underlying thermodynamic solves must be implemented and validated. This paper presents the development of PyCycle's thermodynamics module along with validation results for the analysis and analytic derivatives.

There are a number of different well respected methods for computing accurate thermodynamics properties for air and air-fuel mixtures, many with pre-existing implementations available. NPSS provides a set of different thermodynamics libraries (CEA, JANAF, ALLFUEL, GasTbl), which the user can select from at runtime. ALLFUEL and GasTbl are very computationally efficient, but are based on tabular thermodynamics data and offer lower accuracy. The CEA and JANAF libraries both solve for chemical equilibrium, but the JANAF library only considers a fixed set of species. The $\mathrm{CEA}^{13}$ library is by far the most general, allowing an arbitrary number of user defined chemical species. CEA's generality made it the clear choice amongst the libraries available in NPSS. The major downside to using CEA was that its based on an Fortran implementation that can't easily be augmented with analytic derivatives. One other option was considered, based on Cantera, $\frac{14}{14}$ an object oriented chemical kinetics library that also provides equilibrium calculations. Chin et. al. used Cantera in a PyCycle prototype to predict performance for a two stage compression system, but found it to be very slow $\frac{15}{15}$ Cantera computed equilibrium by running a dynamic simulation until the time derivatives damp out, which can take a larger number of internal iterations. That time-stepping method would also make it significantly more expensive to compute analytic derivatives with. Since neither CEA or Cantera were usable directly, a new thermodynamics implementation was built based on CEA scheme for minimization of Gibbs free energy.

The rest of the paper is organized as follows: a summary of the Gibbs free energy equilibrium equations and the numerical solver used to converge the chemical equilibrium solve are presented in Section III The analysis validation methodology and results are given in Section III. In Section IV numerical validation of the derivatives is presented along with some performance characterization of analytic vs finite difference derivatives. Finally, results from optimizations to find the maximum combustion temperature are presented in Section $\mathrm{V}$ that demonstrate the speed improvements achieved via the use of analytic derivatives. Future work will build on this study to develop the PyCycle propulsion system elements themselves and model a full thermodynamic cycle.

\section{Chemical Equilibrium Equations}

\section{A. Thermodynamic Properties Prediction}

CEA uses a two-step process, represented in Figure 1 to find the thermodynamic state of a gas. First it solves a system of nonlinear equations to minimize Gibbs free energy which gives the equilibrium composition for the gas at the prescribed state. Second an additional set of equations are solved, using the equilibrium composition, to compute the thermodynamic state of the gas. These equations are documented in detail by Gordon and McBride in their seminal paper on CEA but the methods are fundamental to this work ${ }^{13}$ and the key chemical equilibrium equations are reproduced here for completeness. We refer the reader to the original CEA publications for details on the second step, computing the thermodynamic properties from the converged solution.

\section{B. Gibbs Free Energy Minimization}

The full thermodynamic state of a real gas can be defined by any two independent physical state variables (i.e. $T, P, \rho, S, h)$. The CEA method provides calculations for three specific combinations: (1) temperature 
and pressure-TP, (2) enthalpy and pressure- $\mathrm{hP},(3)$ entropy and pressure- $\mathrm{SP}$. The TP solve is the most fundamental. The hP and SP use the same equations as the TP solve augmented with $T$ as a new state variable and an additional residual to drive the solution to the prescribed value of $h$ or $S$.

\section{Temperature-Pressure Solve}

The TP solve is derived as follows:

Gibbs free energy, $g$, is defined as

$$
g=\sum_{j}^{N_{s}}\left(\mu_{j} n_{j}\right),
$$

where $N_{s}$ is the number of chemical species, and $n_{j}$ and $\mu_{j}$ are the concentrations (kg-mol/kg-mixture) and the chemical potential of the $j^{\text {th }}$ species respectively. The chemical potential is a function of temperature $(T)$, pressure $(P)$, and composition $(n)$ given by

$$
\frac{\mu_{j}}{R T}=\frac{H_{j}^{\circ}(T)}{R T}-\frac{S_{j}^{\circ}(T)}{R T}+\ln \left(\frac{P}{P_{a}}\right)+\ln \left(n_{j}\right)-\ln \left(\sum_{k}^{N_{s}} n_{k}\right) .
$$

Both $H^{\circ}$ and $S_{j}^{\circ}$ are functions of $T$ and a number of constants, $c_{0} \ldots c_{8}$, which are given for each species as input to the code. $R$ is the universal gas constant.

$$
\begin{aligned}
\frac{H_{j}^{\circ}(T)}{R T} & =\frac{-c_{0}}{T^{2}}+\frac{c_{1} \ln (T)}{T}+c_{2}+\frac{c_{3}}{2} T+\frac{c_{4}}{3} T^{2}+\frac{c_{5}}{4} T^{3}+\frac{c_{6}}{5} T^{4}+\frac{c_{7}}{T}, \\
\frac{S_{j}^{\circ}(T)}{R} & =\frac{-c_{0}}{2 T^{2}}-\frac{c_{1}}{T}+c_{2} \ln (T)+c_{3} T+\frac{c_{4}}{2} T^{2}+\frac{c_{5}}{3} T^{3}+\frac{c_{6}}{4} T^{4}+c_{8} .
\end{aligned}
$$

Equilibrium composition is defined by the values of the concentration variables, $n$, that minimize Gibbs free energy subject to conservation of mass constraints. Mass is tracked on an elemental basis, given by

$$
\mathcal{R}_{\text {mass } i}=\sum_{j=1}^{N_{s}}\left(a_{i j} n_{j}\right)-b_{i}^{\circ}=0
$$

where $N_{s}$ is the number of species in the mixture; $b_{i}^{\circ}$ is the amount of each element from the initial composition; $a_{i j}$ is the stoichiometric constant for element $i$ of species $j$. Equation (5), defining the mass balance

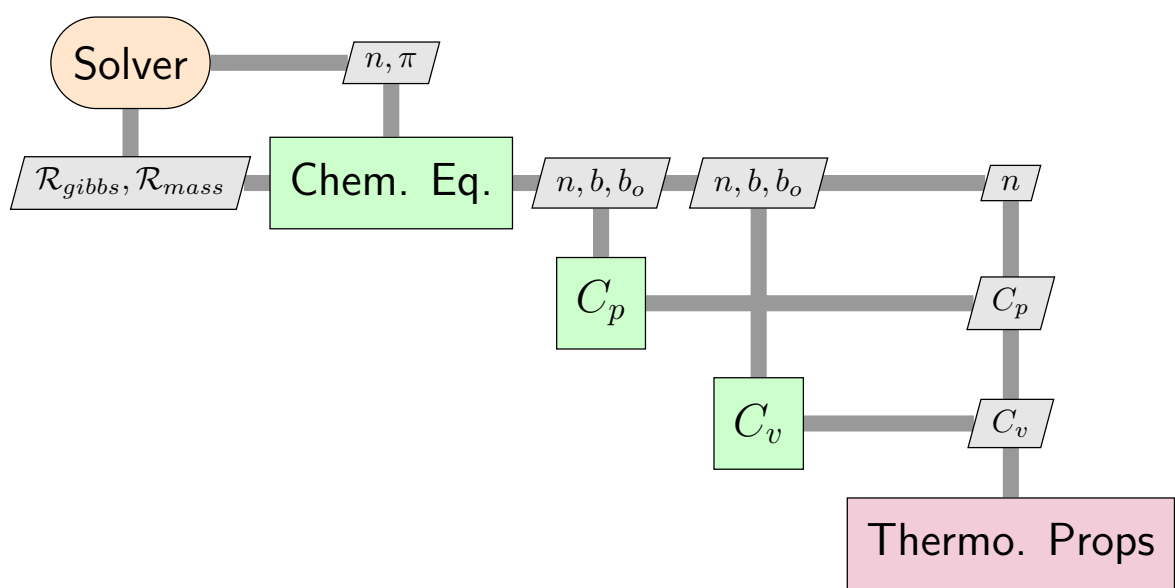

Figure 1: $\operatorname{XDSM}^{16}$ diagram of the two-step chemical equilibrium analysis process. The first step is composed of the nonlinear solve itself, which finds the equilibrium composition vector $n$. The second step uses the converged $n$ values to compute the rest of the thermodynamic properties via explicit functions. 
residuals, yields one constraint per element present in the mixture, giving $N_{e}$ - number of individual elements - constraints. To solve the mass constrained minimization of Gibbs free energy, we form a Lagrangian

$$
G=\sum_{j=1}^{N_{s}}\left(\mu_{j} n_{j}\right)+\sum_{i=1}^{N_{e}} \lambda_{i}\left(\sum_{j=1}^{N_{s}}\left(a_{i j} n_{j}\right)-b_{i}^{\circ}\right)
$$

where $\lambda_{i}$ is the Lagrange multiplier for the $i^{t h}$ element. We take derivatives with respect to the $n$ and $\lambda$ variables to build a system of non-linear equations whose solution will minimize the Lagrangian, as follows:

$$
\delta G=\sum_{j=1}^{n_{s}}\left(\mu_{j}+\sum_{i=1}^{N_{e}}\left(\lambda_{i} a_{i j}\right)\right) \delta n_{j}+\sum_{i=1}^{N_{e}}\left(\sum_{j=1}^{N_{s}}\left(a_{i j} n_{j}\right)-b_{i}^{\circ}\right) \delta \lambda_{i}=0 .
$$

Since $\delta G$ is linear in $\delta n_{j}$ and $\delta \lambda_{i}$, we can split Equation (7) into two sets of equations. We can get $N_{s}$ equations,

$$
\mathcal{R}_{\text {gibbs }}=\mu_{j}+\sum_{i=1}^{N_{e}}\left(\lambda_{i} a_{i j}\right)=0,
$$

as residuals representing Gibbs free energy. For convenience, because of the factors of $1 / R T$ in Equation (2) and Equation (4), we define an alternative Lagrange multiplier as follows:

$$
\pi_{i}=-\frac{\lambda_{i}}{R T}
$$

This gives an alternate form of Equation (8),

$$
\mathcal{R}_{\text {gibbs }}=\frac{\mu_{j}}{R T}-\sum_{i=1}^{N_{e}}\left(\pi_{i} a_{i j}\right)=0 .
$$

Equations (5) and (10) yield a system with $N_{s}+N_{e}$ unknowns and $N_{s}+N_{e}$ residual equations, which can then be converged with a numerical solver.

\section{Enthalpy-Pressure Solve}

The hP solve retains the state variables $(n$ and $\pi$ ) from the TP solve and the associated residuals from Equation (5) and (10). In addition, it adds a new state variable $T$ and a new residual to drive the computed enthalpy to match the the specified value of enthalpy, $h_{0}$ :

$$
\mathcal{R}_{h}=h_{0}-\sum_{j=1}^{N_{s}}\left(n_{j} H_{j}^{\circ}(T)\right) .
$$

\section{Entropy-Pressure Solve}

Like the $\mathrm{hP}$ solve, the $\mathrm{SP}$ uses the same setup as $\mathrm{TP}$ with one additional state variable and residual. In this case, the new state variable is the prescribed entropy, $S_{0}$. The new residual drives the computed entropy to match the perscribed one:

$$
\mathcal{R}_{S}=S_{o}-R \sum_{j=1}^{N_{s}}\left(n_{j}\left(\frac{S_{j}^{\circ}(T)}{R}-\ln \left(\frac{P}{P_{a}}\right)-\ln \left(n_{j}\right)+\ln \left(\sum_{k}^{N_{s}} n_{k}\right)\right)\right) .
$$

Note that the pressure term is non-dimensionalized by pressure at standard atmosphere $\left(P_{a}=1.01325\right.$ Bar). The reference condition is necessary because Entropy is only really defined as a delta from a reference condition. 


\section{CEA Modified Newton's Method for Chemical Equilibrium}

\section{Newton Convergence Scheme}

Gordon and Mcbride applied Newton's method to converge the chemical equilibrium system!13 Netwon's method, applied to the Gibbs free energy minimization, consists of successive solves of the linear system,

$$
\frac{d R}{d U} \Delta U=-R(U)
$$

where $U=(n, \pi)$ for a TP solve and $U=(n, \pi, T)$ for $\mathrm{hP}$ and SP solves. $\Delta U$ is iteratively computed and applied until the residuals given by Equations (5), (10), (11), and $(12)$ converge to withing a given tolerance.

Note that Equation (10) involves computing $\mu_{j}$, which via Equation (2), requires taking the natural $\log$ of $n$. In addition Equation (4) involves taking the natural $\log$ of $T$. Hence it is important that the iterations do not push the values of $n$ or $T$ negative during convergence. A standard Newton's method implementation does not provide a means of ensuring positive values for any of the state variables, and in fact for this problem it commonly produces negative values for $n$ during convergence. To solve this issue, Gordon and Mcbride break up $\Delta U$ into two parts, for $(n, T)$ and $\pi$ variables. They then treat the $(n, T)$ component of the updates as $\Delta \ln (n) / n$ and $\Delta \ln (T) / T$. This modifies the Newton update equation for the $n$ and $T$ variables as follows:

$$
\begin{aligned}
& n_{k+1}=n_{k} \exp \left(\frac{\Delta \ln (n)}{n}\right), \\
& T_{k+1}=T_{k} \exp \left(\frac{\Delta \ln (T)}{T}\right) .
\end{aligned}
$$

By using the exponential form, negative values from the newton solve are converted into multiplicative updates that are always positive, so assuming a positive initial guess the iterations can never push the values negative. This method has a secondary benefit of dramatically improving convergence of the overall model.

The $\pi$ update variables are treated normally, with a standard Newton update equation

$$
\pi_{k+1}=\pi_{k}+\Delta \pi
$$

\section{Computing $d \mathcal{R} / d U$}

$d \mathcal{R} / d U$ is computed analytically, with non-zero elements for the TP solve given by

$$
\begin{gathered}
\frac{d \mathcal{R}_{\text {gibbs } j}}{d n_{k}}= \begin{cases}\frac{-1}{\sum_{l}^{N_{s}} n_{l}} & \text { if } j \neq k \\
\frac{1}{n_{k}}-\frac{-1}{\sum_{l}^{N_{s}} n_{l}} & \text { if } j=k,\end{cases} \\
\frac{d \mathcal{R}_{\text {gibbs } j}}{d \pi_{i}}=-a_{i j}, \\
\frac{d \mathcal{R}_{\text {mass } i}}{d n_{j}}=a_{i j} .
\end{gathered}
$$

When doing an hP or SP solve, additional non-zero derivatives of Equation (8) with respect to $T$ are given by

$$
\frac{d \mathcal{R}_{\text {gibbs } j}}{d T}=\frac{\partial H_{j}^{\circ}}{\partial T}-\frac{\partial S_{j}^{\circ}}{\partial T} .
$$

For an $\mathrm{hP}$ solve, the residual in Equation (11) contributes the following non zero terms:

$$
\begin{gathered}
\frac{d \mathcal{R}_{h}}{d n_{j}}=-T H_{j}^{\circ} \\
\frac{d \mathcal{R}_{h}}{d T}=-T \sum_{j=1}^{N_{s}} n_{j}\left(\frac{\partial H_{j}^{\circ}}{\partial T}+H_{j}^{\circ}\right) .
\end{gathered}
$$


Similarly for an SP solve, the residual in Equation 12 contributes the following non zero terms:

$$
\begin{gathered}
\frac{d \mathcal{R}_{S}}{d n_{j}}=\frac{S_{j}^{\circ}(T)}{R}-\ln \left(\frac{P}{P_{a}}\right)-\ln \left(n_{j}\right)+\ln \left(\sum_{k}^{N_{s}} n_{k}\right)-1, \\
\frac{d \mathcal{R}_{S}}{d T}=\sum_{j=1}^{N_{s}}\left(n_{j} \frac{\partial H_{j}^{\circ}}{\partial T}\right) .
\end{gathered}
$$

All of these non-zero terms can be assembled into a matrix, which is then inverted with a direct method since the size of the matrix is at most $\left(N_{s}+N_{e}+1\right) \times\left(N_{s}+N_{e}+1\right)$, with the largest term $\left(N_{s}\right)$ being on the order of 100 species even for very complex mixtures.

\section{Analysis Validation}

\section{A. Analysis Validation Approach}

The thermodynamics module will serve as the foundation for PyCycle, so it is of paramount importance that it accurately model thermodynamic properties of air and air-fuel mixtures across a wide range of temperatures and pressures. The new code was validated against CEA predictions to ensure accuracy. A regular grid of temperatures and pressures, given in Table 1, were run in both CEA and PyCycle and the results compared at each test point. A total of 3600 different conditions were examined with temperatures ranging from 200 to 4800 degrees Rankine and pressures from 1 to 1500 psi. This grid of test points was run for four comparison cases, with equivalence ratios $(\phi)$ of $0,0.015,0.3$, and 0.44 to provide a wide range of combustion conditions. Equivalence ratio (the ratio of actual fuel-to-air-ratio to the stoichiometric value) is a convenient way to express the amount of potential combustion in a manner that is independent of the specific fuel being used. This test set includes low temperatures that are not physically meaningful, and in fact go below the valid range of the thermodynamic data provided as input. Such low temperatures were run not to test the physical predictive power of the code at invalid conditions, but rather to compare PyCycle and CEA numerically at a far extreme to make sure the two codes handled this situation appropriately. Since PyCycle will be used for optimization, its important the the code return numerically stable results even at silly conditions because optimizations often pass through physically invalid areas on their way to convergence.

Table 1: Regular grid of temperature and pressure conditions used to validate PyCycle against CEA. A total of 3600 test points were run for each of the four $\phi$ settings $0,0.016,0.33$, and 0.5

\begin{tabular}{|r||c|c|c|}
\hline & low & high & step \\
\hline \hline Temperature (degR) & 200 & 4800 & 200 \\
\hline Pressure (PSI) & 1 & 1500 & 10 \\
\hline
\end{tabular}

To compute the validation data, PyCycle was setup with with air at the temperature and pressure conditions prescribed in Table 1 and then combusted at the the given $\phi$ with Jet-A, defined as a hydrocarbon fuel, $\mathrm{C}_{12} \mathrm{H}_{23}$, with a stoichiometric fuel-air ratio of 0.06817 corresponding to $\phi=1$.

For Pycycle the combustion was modeled as an prescribed enthalpy process by computing the overall enthalpy of the gas-fuel combination and holding it constant while solving for a chemical equilibrium composition. Since the total enthalpy calculation was needed for the PyCycle combustion model anyway, the corresponding CEA runs were setup with $h$ and $P$ using the enthalpy output from the corresponding PyCycle case. For both PyCycle and CEA a reduced set of 14 chemical species were considered, in order to reduce the number of trace species present in the converged equilibrium solves and to improve computational speed. The associated CEA input file can be found in Appendix A

Two different types of validation were performed. First, the predicted chemical equilibrium compositions were compared to ensure that the proper amount of each chemical species was present at each test point. Next, the actual thermodynamic properties $\left(P, T, \rho, h, S, C_{p}, C_{v}, \gamma\right)$ were compared. For the composition validation the average discrepancy between PyCycle and CEA was $5.2 e 10^{-6}$ moles and the maximum error was .00011 moles. The average discrepancy in thermodynamic properties prediction was $0.03 \%$ and the 
maximum error was $.52 \%$. These results demonstrate a strong agreement between PyCycle and CEA over a very wide temperature and pressure range.

One unavoidable source of discrepancy between PyCycle and CEA comes from implementation details related to what is done when composition variables, $n_{j}$, trend toward 0 . Taking logarithms and antilogarithms of such small numbers is numerically challenging. CEA establishes a lower limit (user selectable with $1 \mathrm{e}-5$ as the default) below which a chemical species is removed from the equilibrium solve all together and assumed to be 0 .

While this approach has some nice aspects - shrinking the size of the non-linear system and saving the cost of a logarithm evaluations on tiny quantities - it introduces some significant discontinuities into the solution. Discontinuities are problematic for optimization applications because they are a non-differentiable behavior. The result of this effect can be seen clearly in Figure 2 where the number of active species in the converged solution is shown for various different values of $\phi$ over a range of specified temperatures. The number of active species varies the most for $\phi=0$ (from 4 to 11) because at lower temperatures the composition of air stays relatively close to atmospheric, but $N O_{x}$ starts to form and dissociation starts to occur at the higher temperatures. Note that the data in Figure 2 is pressure-averaged over the entires range of pressured from Table 1. There was a very slight negative correlation between pressure and the number of active species in the data, but compared to the temperature effect it was negligible. If a larger set of species were considered, the pressure effect could be more pronounced.

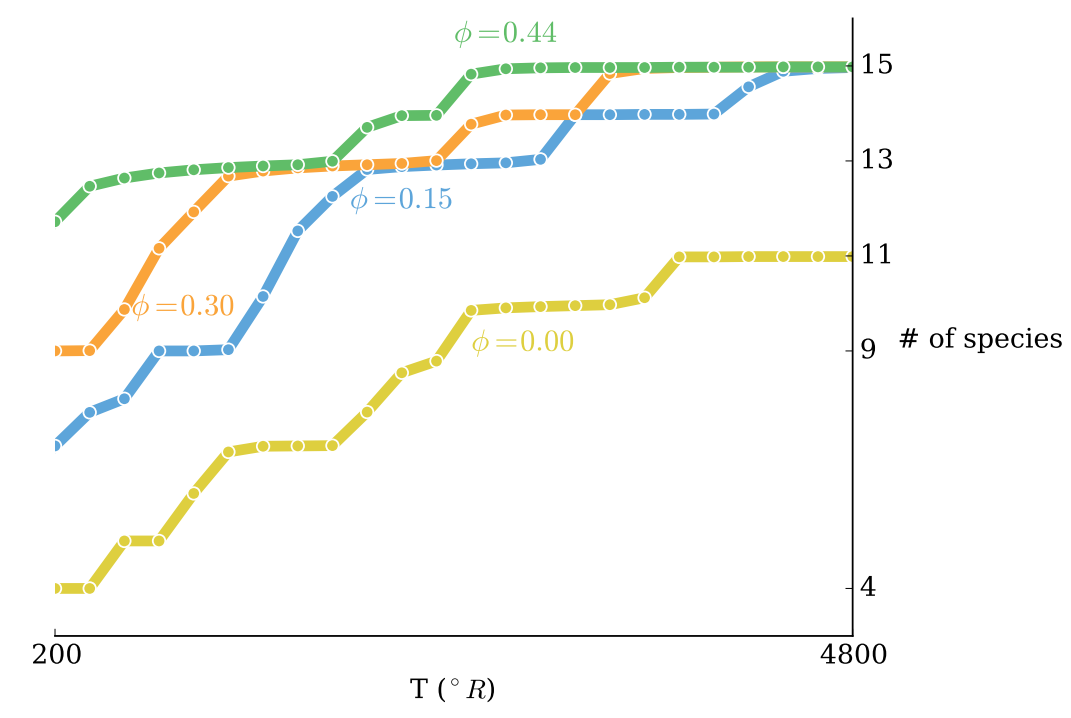

Figure 2: The total number of active chemical species in CEA equilibrium solutions over the test case set from Table 1, for different values of $\phi$. The lower threshold on $n_{j}$, before a species was removed, was set to $1 \mathrm{e}-10$.

The discontinuities from the CEA approach were undesirable for PyCycle, so a a different approach was employed. Instead of removing species from the solution, a numerical limiting was introduced that made it increasingly difficult for the solver to drive composition variable values below the given lower limit - 1e-10 by default. This approach gives a smooth and differentiable response, better suited for the purposes of optimization, but it does introduce a small source of discrepancy between CEA and PyCycle. Since the discrepancy deals with chemical species present only in minuscule amounts, this source of error was deemed more than acceptable.

\section{B. Chemical Equilibrium Validation}

The first validation check performed was to confirm that PyCycle returned the same overall chemical composition as CEA over the prescribed test case range from Table 1. The comparison was done on a per species basis and measured with absolute differences. Air, even when combusted with $\phi=0.44$, is composed of over $70 \%$ diatomic nitrogen. This means that any other chemical species (e.g. $\mathrm{CO}_{2}$ or $\mathrm{H}_{2} \mathrm{O}$ ), while important to the thermodynamic properties make up less than $30 \%$ of of the overall gas. Some species (e.g. $\mathrm{NH}_{3}$, 
$\mathrm{NO}_{3}$ ) are present only in trace amounts and have almost no impact on the thermodynamics. It should be noted that accuracy of compositions of trace species predicted using a chemical equilibrium method is highly limited, but is still of some numerical significance in order to ensure that both PyCycle and CEA predict the same species to be present only in trace amounts. The very low concentrations of these trace species necessitate the use of absolute error to measure the discrepancy between CEA and PyCycle. Absolute error requires knowledge of the actual value for $n_{j}$ in order to be meaningful. Figure 3 shows the mean $n_{j}$ value across the full range of temperatures and pressures as a function of equivalence ratio as the solid blue line. The $\| n_{j}$ CEA $-n_{j}$ PyCycle $\|_{2}$ as an orange curve.

In Figure 3 most of the discrepancies between CEA and PyCycle are at least one order of magnitude smaller than the mean concentrations, indicating very strong agreement between the two codes. For $\mathrm{NH}_{3}$ and $\mathrm{NO}_{3}$ the discrepancies are on the same order of magnitude as the mean value, but given the extremely small levels this did not cause significant changes in the thermodynamic properties. Some discrepancy is expected due to the different methods for handling small concentrations, as discussed above.

In addition to considering the discrepancy of each species individually, Figure 4 examines how the norm of the absolute error, $\left\|n_{\mathrm{CEA}}-n_{\mathrm{PyCycle}}\right\|_{2}$, varies with $T$ and $P$ for the $\phi=0.44$ case. There is a clear correlation between increasing temperature - and to a much weaker extent pressure - and increasing discrepancy between the codes. Although the discrepancy grows from $3 \mathrm{e}-5$ to $2 \mathrm{e}-4$ moving from the lower left to the upper right corners, it is still very small. This trend also corresponds well with Figure 2, where CEA starts introducing small concentrations of new species as the temperature rises. Since CEA and PyCycle use different methods to handle these small amounts of species, some additional discrepancy in areas where this method is most active are expected. The results from Figure 3 and Figure 4 demonstrate that both codes are in strong agreement in terms of overall composition across the entire validation test set. The overall conclusion is that CEA and PyCycle both compute the same composition, within solver tolerance.

The predicted composition vectors will not directly influence Pycycle propulsion elements. The elements will work with the predicted thermodynamic properties of the gas mixture. However its important that CEA and PyCycle agree on predicted composition because the thermodynamic properties are computed as a function of $n$. So the validation of predicted composition serves as a preliminary validation for the thermodynamics themselves. The validation of the thermodynamic properties is presented in the next section, but this data reinforces those results by ensuring that the calculations are fundamentally based on the same chemical compositions.

\section{Thermodynamic Properties Validation}

Comparison of the predicted thermodynamic state variables between CEA and PyCycle was done using relative measurements, since all values have magnitudes larger than 1. Figure 5 shows mean discrepancy, measured across the full test set, for entropy $(S)$, temperature, $(T)$, enthalpy $(h)$, density $(\rho)$, pressure, $(P)$, specific heat ratio $(\gamma)$, and specific heat at constant pressure $\left(C_{p}\right)$. The mean error for all cases, across all properties, was $.03 \%$, and the maximum error was $.52 \%$. Note that since both $h$ and $P$ are set directly in the CEA run cases, from the output of the corresponding PyCycle cases, these properties have the lowest errors in Figure 5. The other errors are larger, but still less than .1\%. This demonstrates an extremely strong agreement between CEA and PyCycle with regard to calculations of the thermodynamic properties. This ensures that PyCycle has a firm thermodynamic foundation upon which cycle modeling elements can be built.

\section{Validating the Multidisciplinary Derivatives}

We define multidisciplinary derivatives as the total derivative taken of the objective of constraints, with respect to the design variables of the model. In other contexts these could also be called total derivatives or coupled derivatives. Admittedly, in this work, there is only a single engineering discipline, thermodynamics. Since the model is built up of components, you can think of the model as being built up from sub-disciplines (e.g. chemical equilibrium and gas properties calculations). In that sense, any derivative taken across an OpenMDAO model as a multidisciplinary derivative. We use "multidisciplinary" to highlight the application, in OpenMDAO, to computing derivatives across a coupled multidisciplinary model. In contrast partial derivatives are taken across a single component (i.e. derivatives of component outputs with respect to its own input variables). OpenMDAO expects these values to be provided to it by components in the models, 

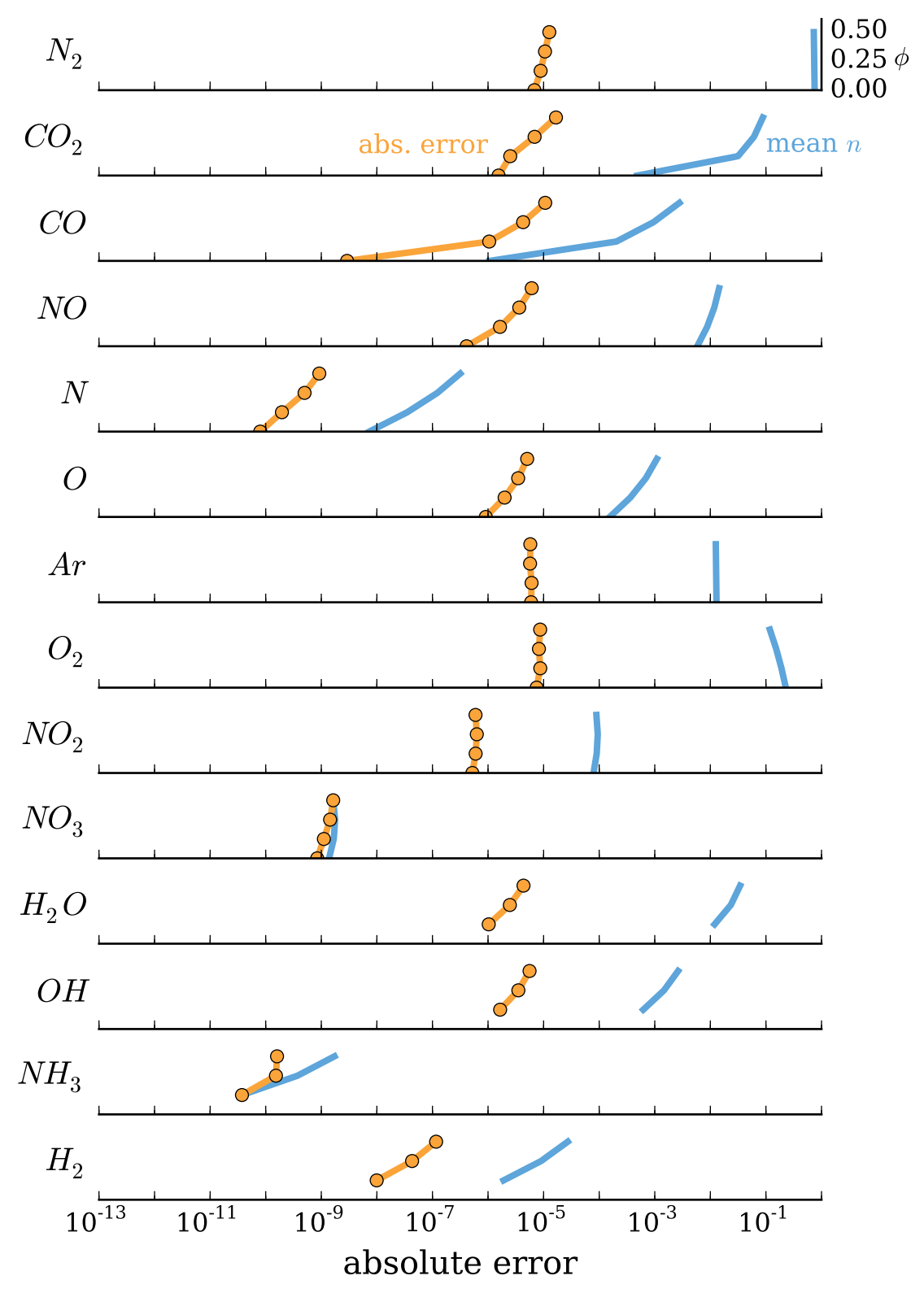

Figure 3: Mean difference between the equilibrium gas composition predicted by CEA and by PyCycle sampled over the test case set from Table 1. The dashed, blue line indicate the value of the mean composition for each species to give a comparison between the magnitude of the error and the amount of species present.

or it can approximate their values with finite-differences. Either way, these values are needed in order to compute the multidisciplinary derivatives. Given the partials the multidisciplinary derivatives are then computed by OpenMDAO by solving a linear system which accounts for all the data connections and coupling between components.

Since the motivation of this work was ultimately to build a tool well suited to gradient based optimization, derivative accuracy is just as important as analysis accuracy. This section presents the results of derivatives comparisons between the multidisciplinary derivatives and finite-difference approximations. Figure 6 shows $\log -\log$ plots of finite-difference derivative error vs step-size for derivatives of $h, S, \rho, C_{p}, \gamma$ with respect to $T$ and $P$, taken at standard day conditions. Each row represents derivatives of one response variable, and each column represents derivatives with respect to one independent variable. Strong agreement, with errors 


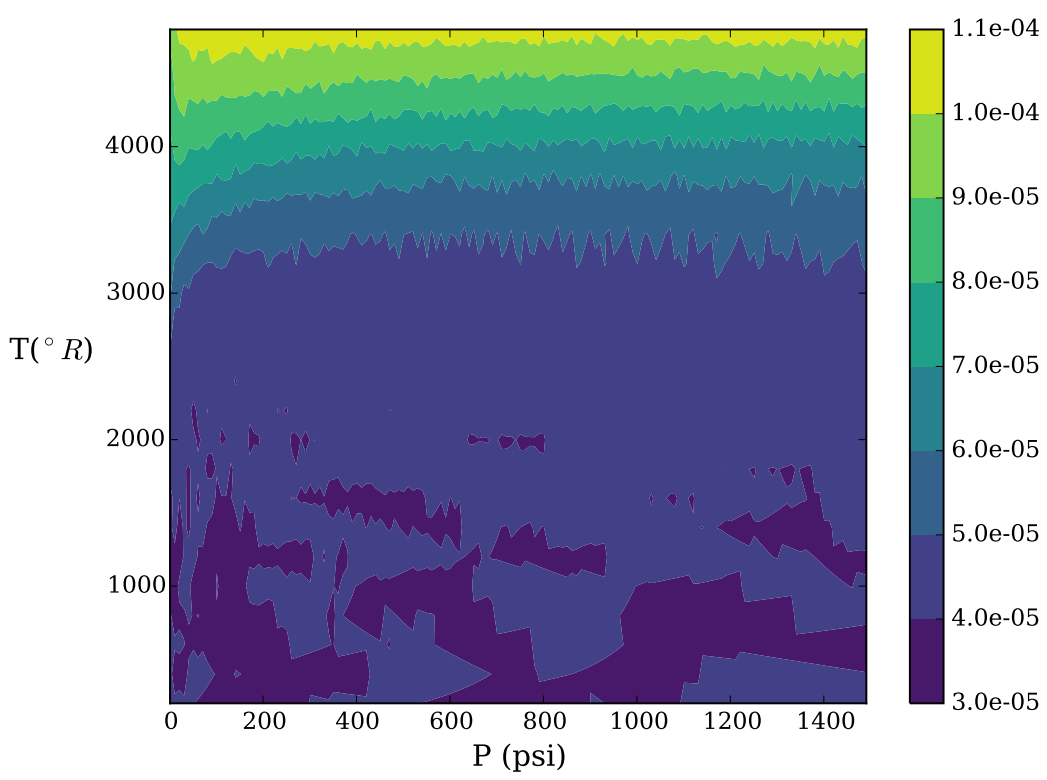

Figure 4: $\left\|n_{\mathrm{CEA}}-n_{\mathrm{PyCycle}}\right\|_{2}$ for $\phi=.44$. This represents overall discrepancy between CEA and Pycycle at any point. There is clear trend for increasing - though still small - discrepancy as higher temperatures, and a much weaker correlation with increasing pressure.

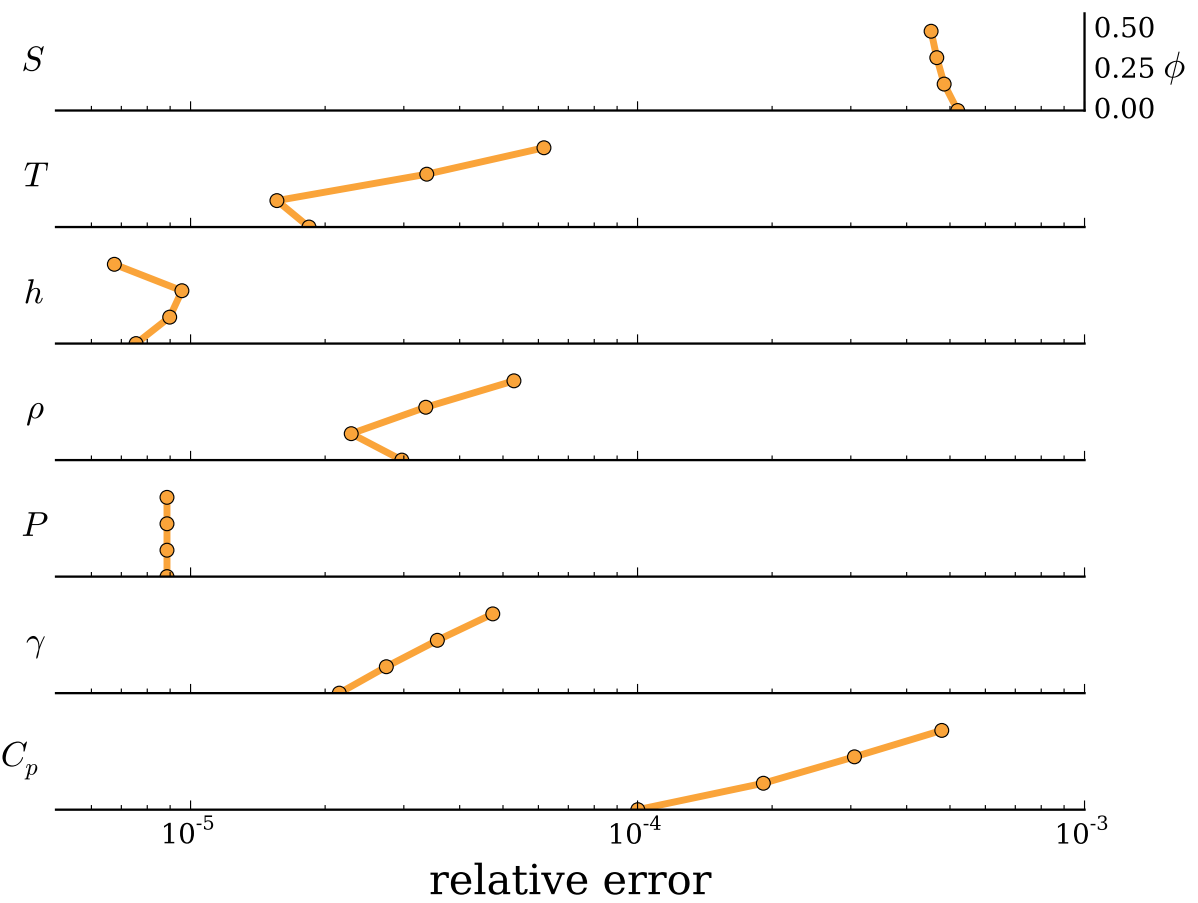

Figure 5: Mean relative error between the thermodynamics states predicted by CEA and by PyCycle sampled over the test case set from table 1 . 


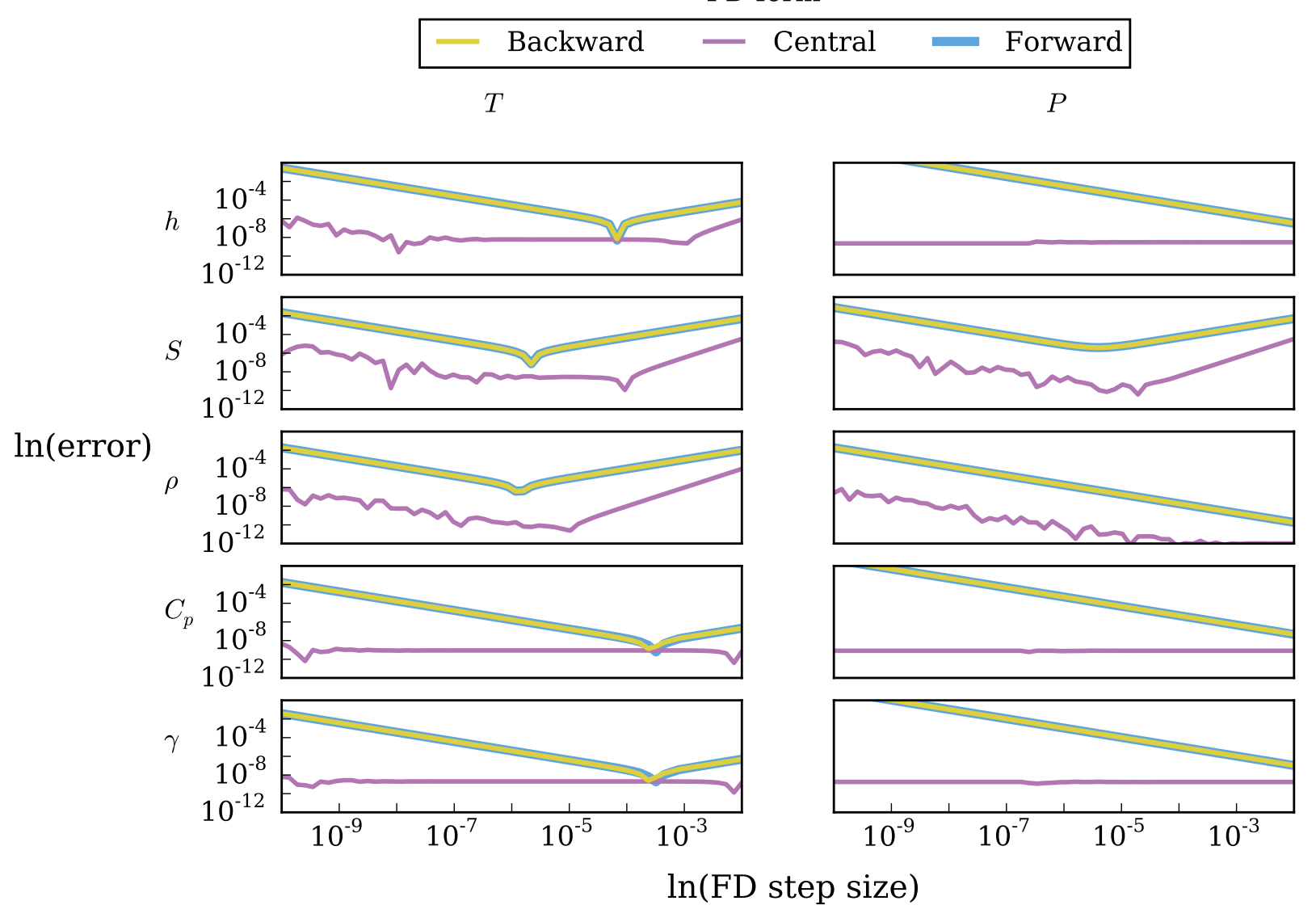

Figure 6: Finite-difference error for derivatives of $h, S, \rho, C_{p}, \gamma$ with respect to $T$ and $P$, taken at standard day conditions. The data is presented in a Jacobian style format, with a single row for each response and column for each independent variable. Errors below $10^{-10}$ were achieved at various step sizes. Notably, minimum error was found at different step sizes for different combinations of variables.

below $10^{-10}$, were achieved all of the derivatives at different step sizes using the central difference scheme.

In Figure 66, the central difference scheme converges faster than the forward or backward schemes, and overall offers better accuracy over of a range of step sizes. This is expected, since central differencing is $2^{\text {nd }}$ order accurate compared to $1^{\text {st }}$ order accurate for the other two schemes. This accuracy is not free, as central differencing requires two function evaluations for each derivative. This compute cost shows up clearly in Figure 7, which presents the compute times for various methods to assemble the full Jacobian of design variables and responses represented in Figure 6. The central difference scheme has twice the compute cost of the right and left difference schemes. Analytic derivatives are faster then the FD schemes, with the forward analytic method being the fastest. Each finite difference step requires the convergence of the full nonlinear model, however OpenMDAO computes the analytic derivatives using only linear solves via Martins and Hwang's unified derivatives equations.17 The additional cost of the adjoint vs the forward analytic derivatives method has to do with the number of linear solves for required for each one. There were 2 independent variables, and 5 responses. So for forward mode, 2 linear solves were needed. For adjoint mode 5 were needed. With larger design spaces the adjoint method would be faster because the design variables far out number the number objective and constraints. 


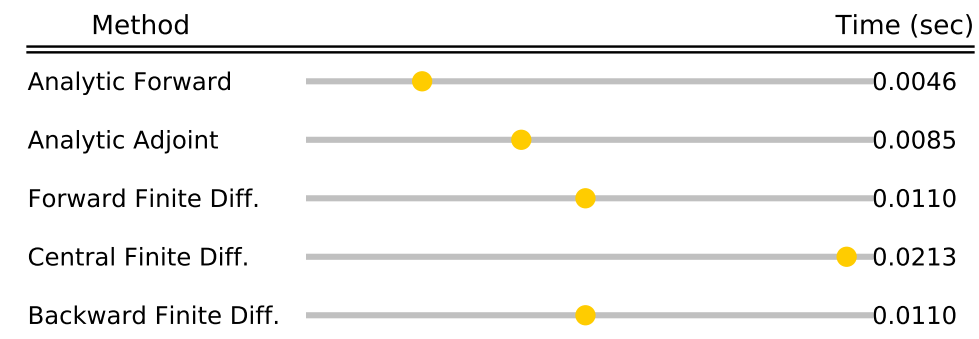

Figure 7: Wall times required to compute a full Jacobian for derivatives of $h, S, \rho, C_{p}, \gamma$ with respect to $T$ and $P$. All analytic methods were faster than the finite-difference methods with forward analytic being the fastest because there were 2 design variables and 5 responses. All of the compute times were generated on an Apple Macbook laptop with a 2.6 Ghz dual-core Intel Core i5 processor and $16 \mathrm{~Gb}$ of memory.

\section{Optimizations}

\section{A. Equivalence Ratio Optimization}

We ran a series of unconstrained optimizations at a fixed gas temperature of $518^{\circ} R$, varying $\phi$ to maximize combustion temperature across different pressures from 15 psi to 1500 psi. Figure 8 shows the results of the optimizations. Somewhat counter-intuitively, the maximum temperature occurs for $\phi$ between 1.07 and 1.02. Under the assumption of perfect combustion, the maximum temperature would occur at stoichiometric, $\phi=1$, with every molecule of diatomic oxygen being converted to water. However equilibrium calculations take into account disassociation effects, which simultaneously lowers the maximum achievable temperature and causes that temperature to occur at a richer $\phi ! \frac{18}{18}$ The effect of dissociation becomes less severe at higher pressures because they tend to favor the creation of slightly larger molecules and the reaction more closely approximates ideal combustion. In Figure 8 this manifests in both the increasing maximum temperature and the decreasing optimal $\phi$ value as pressure increases. These results were run with both finite difference and analytic derivatives, but the results were essentially identical both in terms of overall execution time and final objective value. For this simple, one design variable, case analytic derivatives don't provide significant speed improvements or accuracy advantage.

These results establish that the best conditions for combustion, even accounting for equilibrium chemistry effects, are higher pressures. Therefore, one would expect that adding pressure as a design variable would yield the same result without the need to perform the parameter study across pressure.

\section{B. Equivalence Ratio \& Pressure Optimization}

To verify this a second series of optimizations were run with both $\phi$ and $P$ as design variables, again seeking to maximize combustion temperature to test this behavior. For this set of runs, the numerical solver tolerance for the chemical equilbrium was varied from 1e- 8 to $1 \mathrm{e}-12$ to test the sensitivity of the different gradient methods to depth of convergence. Both derivative methods were able to recover the expected result of $\phi=1.021$ and $P=1500$ (psi). However, unlike the single design variable run, the analytic method provided a clear performance advantage over the finite-difference method. The finite-difference approximation was at least twice as expensive as the adjoint analytic method, as shown in Figure 9. One of the main challenges with using finite difference derivatives is the need to have tight tolerances set on any numerical solvers, such as the one used to converge chemical equilibrium. The data in Figure 9 quantifies this effect by comparing performance across different chemical equilibrium solver tolerances. The analytic derivatives give a nearly constant computation time. However the finite-difference take between 5.5 seconds and 110 seconds depending on solver tolerance and finite-difference step size. The wide range in compute times are a result of inaccurate derivatives forcing the optimizer to take more iterations to find the correct answer. Significantly, the behavior for the 1e-6 step size is not monotonic with respect to solver tolerance either. The worst performance came when using a step size of $1 \mathrm{e}-6$ with tolerances between 1e-11 and 1e-10, but the times dropped back down for step sizes between 1e-10 and 1e-9. This indicates a high degree of numerical instability in the finite difference derivatives. For both step-sizes, at a solver tolerance of 1e-8 the compute 


\section{Optimal $\phi \&$ Temperature vs Pressure}
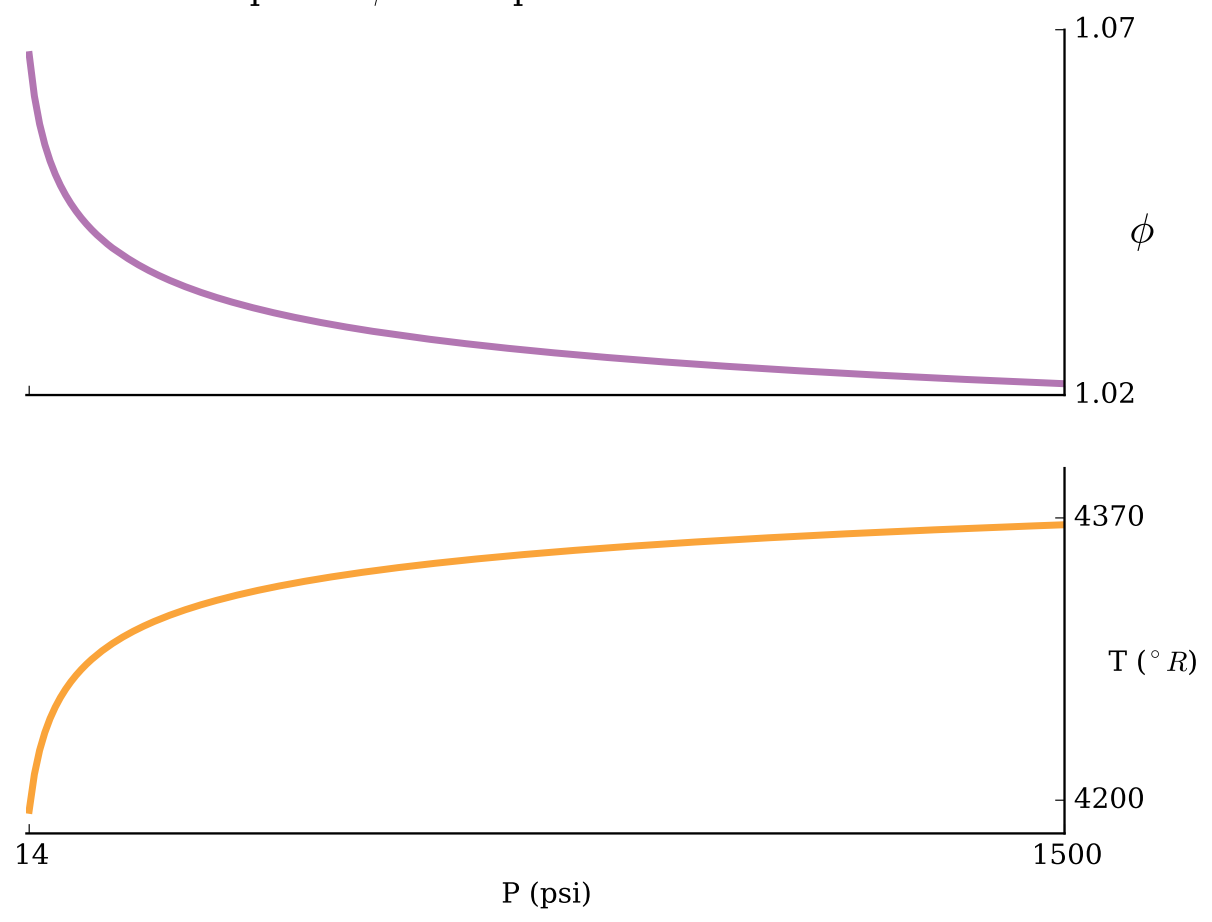

Figure 8: Optimization of Equivalence Ratio, $\phi$, to find maximum combustion temperature at a range of different pressures. Note that maximum temperature is reached at a slightly rich $\phi$ due to dissociation affects, which become less pronounced at higher pressures.

times started to rise. Beyond that point, numerical noise prevented the optimizer from converging using the finite difference derivatives. This result clearly highlights the value of the analytic approach. Even for just 2 design variables, the analytic derivatives provide both a significantly faster and more stable optimization.

\section{Conclusion}

The growing need to incorporate propulsion cycle modeling into multidisciplinary optimizations for aircraft design, which rely on gradient based optimization methods and adjoint analytic derivatives, has motivated the development of a new propulsion cycle modeling tool, PyCycle. In order to develop PyCycle it was first necessary to build the core thermodynamics modeling capability, because that is a central module that enables building the cycle components such as a compressor, combustor, or turbine. This paper presents the development and validation of this thermodynamics module, based on an equilibrium chemistry method originally developed for the CEA code. Since of the calculations for propulsion system elements revolve around the thermodynamic calculations it was essential that both the analysis and derivative calculations of this module be heavily validated. The accuracy of the analysis was validated against the original CEA code and overall results agreed very well across a range of temperature and pressure conditions and at different equivalence ratios. The analytic derivatives were first validated against finite difference approximations and then further validated via usage in sample optimizations.

From the derivative validation data and sample optimization runs, performance comparisons were made between finite difference and analytic methods of computing multidisciplinary derivatives. The analytic derivatives were significantly faster than the finite difference methods, even for a two variable optimization maximizing combustion temperature by varying pressure and equivalence ratio. In addition, the analytic derivatives made the performance of the optimization much less sensitive to the tolerance of the numerical solver for the chemical equilibrium calculations. The improvements in speed and accuracy clearly demonstrate the value of the analytic derivatives approach and provide solid motivation for the continued development of propulsion elements, analogous to those in NPSS, for Pycycle. 


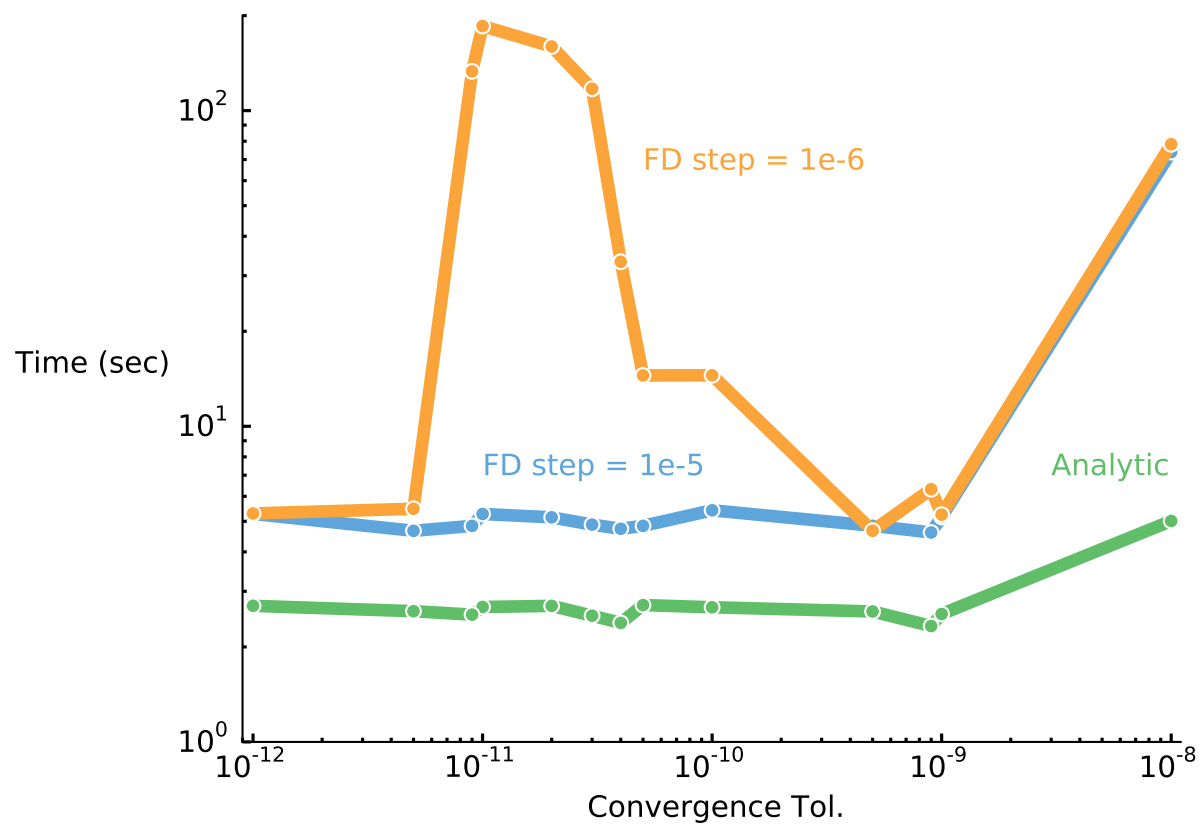

Figure 9: Optimization performance as a function of solver tolerance for the chemical equilibrium solve. The green line shows performance of the analytic derivatives method. The blue and orange lines show performance of the finite-difference derivatives for step-sizes of 1 e-5 and 1e-6 respectively.

\section{Acknowledgments}

We like to thank Dr. Kenneth Moore and Bret Naylor for their technical support in developing needed features in the OpenMDAO framework to enable this work and for helping to debug some of the more tricky analytic derivatives. The authors would also like to thank the NASA Transformational Tools and Technologies Project, under the Aeronautics Research Mission Directorate, for providing funding for this work. 


\section{References}

${ }^{1}$ Hooker, J. R., Wick, A., Zeune, C., and Agelastos, A., "Over Wing Nacelle Installations for Improved Energy Efficiency," 31st AIAA Applied Aerodynamics Conference, June 2013, pp. AIAA 2013-2920.

${ }^{2}$ Fujino, M. and Kawamura, Y., "Wave-drag characteristics of an over-the-wing nacelle business-jet configuration," Journal of Aircraft, Vol. 40, No. 6, 2003, pp. 1177-1184.

${ }^{3}$ Felder, J. L., Kim, H. D., and Brown, G. V., "Turboelectric distributed propulsion engine cycle analysis for hybrid-wingbody aircraft," 47th AIAA Aerospace Sciences Meeting, Orlando, FL, January, 2009.

${ }^{4}$ Nielsen, E. J. and Diskin, B., "Discrete Adjoint-Based Design for Unsteady Turbulent Flows on Dynamic Overset Unstructured Grids," AIAA Journal, Vol. 51, No. 6, June 2013, pp. 1355-1373.

${ }^{5}$ Palacios, F., Economon, T. D., Aranake, A. C., Copeland, S. R., Lonkar, A. K., Lukaczyk, T. W., Manosalvas, D. E., Naik, K. R., Padrón, A. S., Tracey, B., et al., "Stanford University Unstructured (SU2): Open-source analysis and design technology for turbulent flows," AIAA paper, Vol. 243, 2014, pp. 13-17.

${ }^{6}$ Kenway, G. K. W., Kennedy, G. J., and Martins, J. R. R. A., "Scalable parallel approach for high-fidelity steady-state aeroelastic analysis and adjoint derivative computations," AIAA Journal, Vol. 52, 2014, pp. 935-951.

${ }^{7}$ Jones, S., An Introduction to Thermodynamic Performance Analysis of Aircraft Gas Turbine Engine Cycles Using the Numerical Propulsion System Simulation Code, NASA, 2007, TM-2007-214690.

${ }^{8}$ Allison, D. L., Morris, C. C., Schetz, J. A., Kapania, R. K., Sultan, C., Watson, L. T., Deaton, J. D., and Grandhi, R. V., "A Multidisciplinary Design Optimization Framework for Design Studies of an Efficient Supersonic Air Vehicle," 12th AIAA Aviation Technology, Integration, and Operations (ATIO) Conference and 14th AIAA/ISSMO Multidisciplinary Analysis and Optimization Conference, 2012, pp. AIAA 2012-5492.

${ }^{9}$ Allison, D. L., Alyanak, E., and Bhagat, N., "High Fidelity, Nonlinear, Integrated Nozzle Installation Effects for Numerical Propulsion System Simulation," 56th AIAA/ASCE/AHS/ASC Structures, Structural Dynamics, and Materials Conference, 2015, pp. AIAA 2015-0649.

${ }^{10}$ Geiselhart, K. A., Ozoroski, L. P., Fenbert, J. W., Shields, E. W., and Li, W., "Integration of multifidelity multidisciplinary computer codes for design and analysis of supersonic aircraft," 49th AIAA Aerospace Sciences Meeting, No. 2011-465, 2011.

${ }^{11}$ Hendricks, E. S., Jones, S. M., and Gray, J. S., "Design Optimization of a Variable-Speed Power-Turbine," $50 T H$ AIAA/ASME/SAE/ASEE Joint Propulsion Conference, AIAA, Cleveland, Ohio, July 2014, AIAA-2014-3445.

${ }^{12}$ Gray, J. S., Hearn, T. A., Moore, K. T., Hwang, J., Martins, J., and Ning, A., "Automatic Evaluation of Multidisciplinary Derivatives Using a Graph-Based Problem Formulation in OpenMDAO," 15th AIAA/ISSMO Multidisciplinary Analysis and Optimization Conference, American Institute of Aeronautics and Astronautics, 2014/07/08 2014.

${ }^{13}$ Gordon, S. and McBride, B. J., Computer program for calculation of complex chemical equilibrium compositions, rocket performance, incident and reflected shocks, and Chapman-Jouguet detonations, NASA, 1994, NASA RP-1311.

${ }^{14}$ Goodwin, D. G., Moffat, H. K., and Speth, R. L., "Cantera: An Object-oriented Software Toolkit for Chemical Kinetics, Thermodynamics, and Transport Processes," http://www.cantera.org, 2014, Version 2.1.2.

${ }^{15}$ Chin, J., Gray, J., Jones, S., and Berton, J., "Open-Source Conceptual Sizing Models for the Hyperloop Passenger Pod," 2015 AIAA SciTech Conference, American Institute of Aeronautics and Astronautics, Janurary 2015.

${ }^{16}$ Lambe, A. B. and Martins, J. R. R. A., "Extensions to the Design Structure Matrix for the Description of Multidisciplinary Design, Analysis, and Optimization Processes," Structural and Multidisciplinary Optimization, Vol. 46, 2012, pp. 273-284.

${ }^{17}$ Martins, J. R. R. A. and Hwang, J. T., "Review and Unification of Methods for Computing Derivatives of Multidisciplinary Computational Models," AIAA Journal, Vol. 51, No. 11, November 2013, pp. 2582-2599.

${ }^{18}$ Lefebvre, A. H., Gas turbine combustion, CRC press, 3rd ed., 1998. 


\section{Appendix A CEA Species Definition}

SIX-CHARACTER REFERENCE-DATE CODES

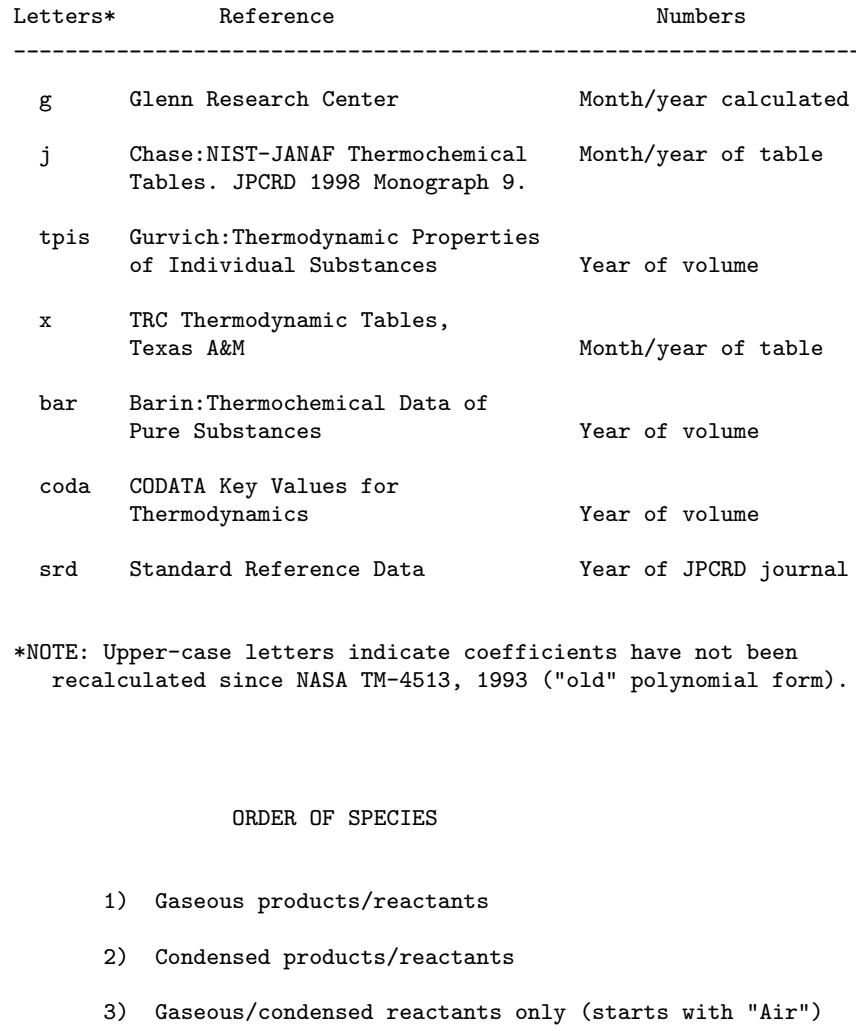




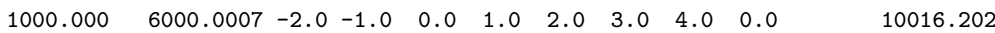
$3.730042760 \mathrm{D}+06-1.383501485 \mathrm{D}+04 \quad 2.049107091 \mathrm{D}+01-1.961974759 \mathrm{D}-03 \quad 4.727313040 \mathrm{D}-07$ $-3.728814690 \mathrm{D}-11 \quad 1.623737207 \mathrm{D}-15$. C2H4 TRC w2600 4/88. JPCRD 1975 v4 p251. Chem Phys 1985 v98 p1.

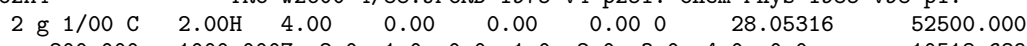

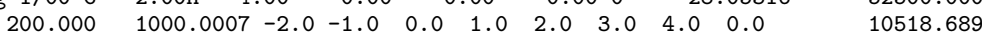

-1.163605836D+05 2.554851510D+03-1.609746428D+01 6.625779320D-02-7.885081860D-05

$5.125224820 \mathrm{D}-08-1.370340031 \mathrm{D}-11 \quad-6.176191070 \mathrm{D}+03 \quad 1.093338343 \mathrm{D}+02$

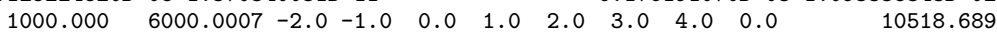

$3.408763670 \mathrm{D}+06-1.374847903 \mathrm{D}+04 \quad 2.365898074 \mathrm{D}+01-2.423804419 \mathrm{D}-03 \quad 4.431395660 \mathrm{D}-07$ $-4.352683390 \mathrm{D}-11 \quad 1.775410633 \mathrm{D}-15 \quad 8.820429380 \mathrm{D}+04-1.371278108 \mathrm{D}+02$ IC2H4 TRC w2600 4/88. JPCRD 1975 v4 p251. Chem Phys 1985 v98 p1.

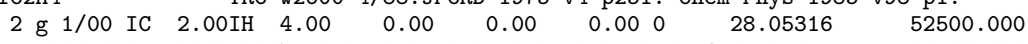

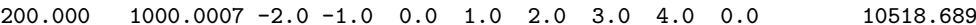

$\begin{array}{llll}-1.163605836 \mathrm{D}+05 & 2.554851510 \mathrm{D}+03-1.609746428 \mathrm{D}+01 & 6.625779320 \mathrm{D}-02-7.885081860 \mathrm{D}-05\end{array}$

$5.125224820 \mathrm{D}-08-1.370340031 \mathrm{D}-11 \quad-6.176191070 \mathrm{D}+03 \quad 1.093338343 \mathrm{D}+02$

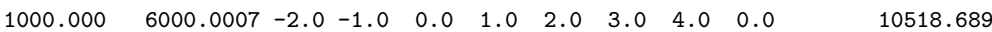

$3.408763670 \mathrm{D}+06-1.374847903 \mathrm{D}+04 \quad 2.365898074 \mathrm{D}+01-2.423804419 \mathrm{D}-03 \quad 4.431395660 \mathrm{D}-07$ $\begin{array}{lll}-4.352683390 \mathrm{D}-11 & 1.775410633 \mathrm{D}-15 & 8.820429380 \mathrm{D}+04-1.371278108 \mathrm{D}+02\end{array}$ C10H8, naphthale Naphthalene. Chen, JPCFD v8 n2 1979 p527-535.

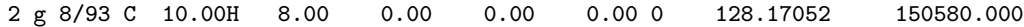

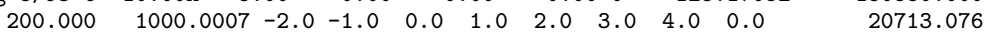

$\begin{array}{lll}-2.602920990 \mathrm{D}+05 & 6.237519290 \mathrm{D}+03-5.226157400 \mathrm{D}+01 & 2.397710630 \mathrm{D}-01-2.912272160 \mathrm{D}-04\end{array}$

$\begin{array}{llll}1.854965710 \mathrm{D}-07-4.816685340 \mathrm{D}-11 & -1.114753250 \mathrm{D}+04 & 2.972172708 \mathrm{D}+02\end{array}$

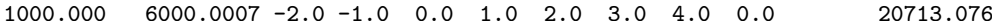

$5.905864570 \mathrm{D}+06-3.163144910 \mathrm{D}+04 \quad 7.030252590 \mathrm{D}+01-6.018395960 \mathrm{D}-03 \quad 1.141923860 \mathrm{D}-06$ $\begin{array}{lll}-1.161432010 \mathrm{D}-10 & 4.891928210 \mathrm{D}-15 & 1.962512700 \mathrm{D}+05-4.347785692 \mathrm{D}+02\end{array}$ IC10H8, naphthal Naphthalene. Chen, JPCFD v8 n2 1979 p527-535.

$\begin{array}{llllllll}2 \text { g } 8 / 93 \text { IC } 10.00 \text { IH } & 8.00 & 0.00 & 0.00 & 0.00 & 0 & 128.17052 & 150580.000\end{array}$

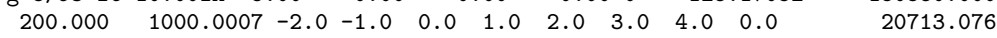

$\begin{array}{llll}-2.602920990 D+05 & 6.237519290 D+03-5.226157400 D+01 & 2.397710630 D-01-2.912272160 D-04\end{array}$

$1.854965710 \mathrm{D}-07-4.816685340 \mathrm{D}-11 \quad-1.114753250 \mathrm{D}+042.972172708 \mathrm{D}+02$

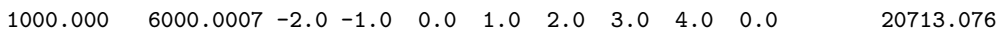
$5.905864570 \mathrm{D}+06-3.163144910 \mathrm{D}+04 \quad 7.030252590 \mathrm{D}+01-6.018395960 \mathrm{D}-03 \quad 1.141923860 \mathrm{D}-06$ -1.161432010D-10 4.891928210D-15 1.962512700D+05-4.347785692D+02

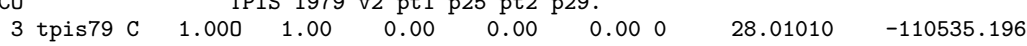

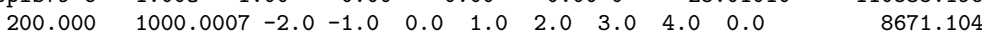

$1.489045326 \mathrm{D}+04-2.922285939 \mathrm{D}+02 \quad 5.724527170 \mathrm{D}+00-8.176235030 \mathrm{D}-03 \quad 1.456903469 \mathrm{D}-05$

$\begin{array}{lll}-1.087746302 \mathrm{D}-08 & 3.027941827 \mathrm{D}-12 & -1.303131878 \mathrm{D}+04-7.859241350 \mathrm{D}+00\end{array}$

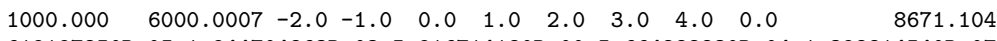
$4.619197250 \mathrm{D}+05-1.944704863 \mathrm{D}+03 \quad 5.916714180 \mathrm{D}+00-5.664282830 \mathrm{D}-04 \quad 1.398814540 \mathrm{D}-07$

$-1.787680361 \mathrm{D}-119.620935570 \mathrm{D}-16 \quad-2.466261084 \mathrm{D}+03-1.387413108 \mathrm{D}+01$

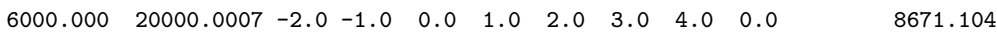
$8.868662960 \mathrm{D}+08-7.500377840 \mathrm{D}+05$
$2.495474979 \mathrm{D}+02-3.956351100 \mathrm{D}-02 \quad 3.297772080 \mathrm{D}-06$ $-1.318409933 \mathrm{D}-101.998937948 \mathrm{D}-15 \quad 5.701421130 \mathrm{D}+06-2.060704786 \mathrm{D}+03$

$\begin{array}{lllllllll}3 \mathrm{~g} 9 / 99 \mathrm{C} & 1.000 & 2.00 & 0.00 & 0.00 & 0.00 & 0 & 44.00950 & -393510.000\end{array}$

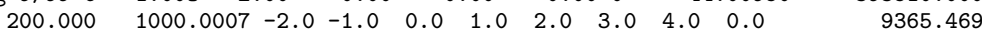

4.943650540D+04-6.264116010D+02 5.301725240D+00 2.503813816D-03-2.127308728D-07

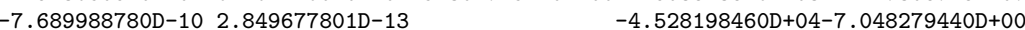

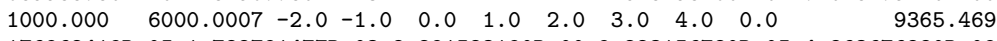
$1.176962419 \mathrm{D}+05-1.788791477 \mathrm{D}+03 \quad 8.291523190 \mathrm{D}+00-9.223156780 \mathrm{D}-05 \quad 4.863676880 \mathrm{D}-09$

$-1.891053312 \mathrm{D}-126.330036590 \mathrm{D}-16 \quad-3.908350590 \mathrm{D}+04-2.652669281 \mathrm{D}+01$

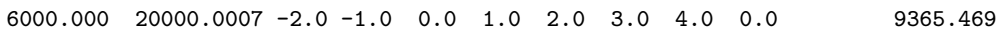
-1.544423287D+09 1.016847056D+06-2.561405230D+02 3.369401080D-02-2.181184337D-06 6.991420840D-11-8.842351500D-16 -8.043214510D+06 2.254177493D+03

$\begin{array}{lllllllll}3 \mathrm{~g} 6 / 97 \mathrm{H} & 1.00 & 0.00 & 0.00 & 0.00 & 0.00 & 0 & 1.00794 & 217998.828\end{array}$

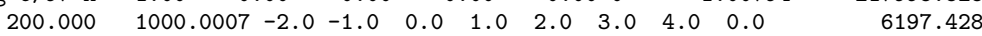
$\begin{array}{lllll}0.000000000 D+00 & 0.000000000 D+00 & 2.500000000 D+00 & 0.000000000 D+00 & 0.000000000 D+00\end{array}$ $0.000000000 \mathrm{D}+00 \quad 0.000000000 \mathrm{D}+00 \quad 2.547370801 \mathrm{D}+04-4.466828530 \mathrm{D}-01$

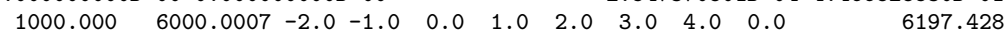
$6.078774250 \mathrm{D}+01-1.819354417 \mathrm{D}-01 \quad 2.500211817 \mathrm{D}+00-1.226512864 \mathrm{D}-07 \quad 3.732876330 \mathrm{D}-11$

$\begin{array}{lll}-5.687744560 \mathrm{D}-15 & 3.410210197 \mathrm{D}-19 & 2.547486398 \mathrm{D}+04-4.481917770 \mathrm{D}-01\end{array}$

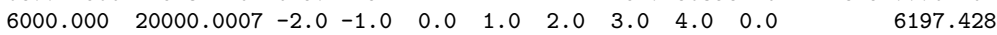
$2.173757694 \mathrm{D}+08-1.312035403 \mathrm{D}+05 \quad 3.399174200 \mathrm{D}+01-3.813999680 \mathrm{D}-03 \quad 2.432854837 \mathrm{D}-07$ -7.694275540D-12 9.644105630D-17 1.067638086D+06-2.742301051D+02 IH DO(H2): JMolSpc, v33 1970 p147. NSRDS-NBS 3 SEC 61972.

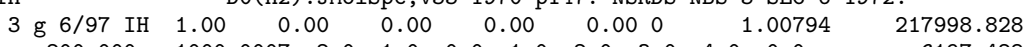

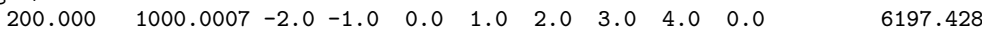
$\begin{array}{llllll}0.000000000 D+00 & 0.000000000 D+00 & 2.500000000 D+00 & 0.000000000 D+00 & 0.000000000 D+00\end{array}$ $0.000000000 \mathrm{D}+00 \quad 0.000000000 \mathrm{D}+00 \quad 2.547370801 \mathrm{D}+04-4.466828530 \mathrm{D}-01$

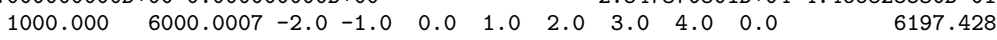

$6.078774250 \mathrm{D}+01-1.819354417 \mathrm{D}-01 \quad 2.500211817 \mathrm{D}+00-1.226512864 \mathrm{D}-07 \quad 3.732876330 \mathrm{D}-11$

$\begin{array}{lll}-5.687744560 \mathrm{D}-15 & 3.410210197 \mathrm{D}-19 & 2.547486398 \mathrm{D}+04-4.481917770 \mathrm{D}-01\end{array}$

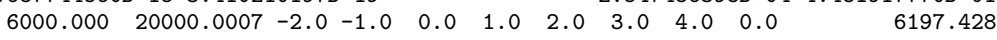
$2.173757694 \mathrm{D}+08-1.312035403 \mathrm{D}+05 \quad 3.399174200 \mathrm{D}+01-3.813999680 \mathrm{D}-03 \quad 2.432854837 \mathrm{D}-07$ $-7.694275540 \mathrm{D}-12 \quad 9.644105630 \mathrm{D}-17 \quad 1.067638086 \mathrm{D}+06-2.742301051 \mathrm{D}+02$ HO2 Hills JCP 1984 v81 p4458. Jacox JPCRD 1988 v17 p303.

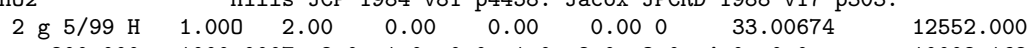

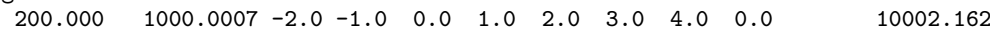
$\begin{array}{lll}-7.598882540 \mathrm{D}+04 & 1.329383918 \mathrm{D}+03-4.677388240 \mathrm{D}+00 & 2.508308202 \mathrm{D}-02-3.006551588 \mathrm{D}-05\end{array}$ 
$1.895600056 \mathrm{D}-08-4.828567390 \mathrm{D}-12 \quad-5.809366430 \mathrm{D}+03 \quad 5.193602140 \mathrm{D}+01$

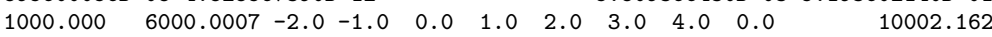
$-1.810669724 \mathrm{D}+064.963192030 \mathrm{D}+03-1.039498992 \mathrm{D}+00 \quad 4.560148530 \mathrm{D}-03-1.061859447 \mathrm{D}-06$ $1.144567878 \mathrm{D}-10-4.763064160 \mathrm{D}-15 \quad-3.194418740 \mathrm{D}+04 \quad 4.066850920 \mathrm{D}+01$ $\mathrm{H} 2$

Ref-Elm. TPIS 1978 v1 pt1 p107 pt2 p31.

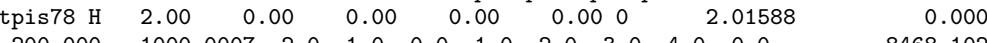
$\begin{array}{lllllllllll}1000.0007 & -2.0 & -1.0 & 0.0 & 1.0 & 2.0 & 3.0 & 4.0 & 0.0 & 8468.102\end{array}$ $4.078322810 \mathrm{D}+04-8.009185450 \mathrm{D}+02 \quad 8.214701670 \mathrm{D}+00-1.269714360 \mathrm{D}-02 \quad 1.753604930 \mathrm{D}-05$ $\begin{array}{lll}-1.202860160 \mathrm{D}-08 & 3.368093160 \mathrm{D}-12 & 2.682484380 \mathrm{D}+03-3.043788660 \mathrm{D}+01\end{array}$

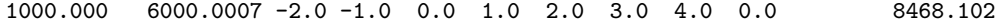
$5.608123380 \mathrm{D}+05-8.371491340 \mathrm{D}+02 \quad 2.975363040 \mathrm{D}+00 \quad 1.252249930 \mathrm{D}-03-3.740718420 \mathrm{D}-07$

$5.936628250 \mathrm{D}-11-3.606995730 \mathrm{D}-15 \quad 5.339815850 \mathrm{D}+03-2.202764050 \mathrm{D}+00$

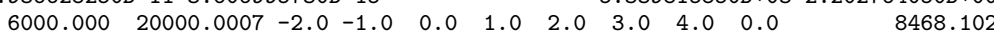
4.966716130D+08-3.147448120D+05 7.983887500D+01-8.414504190D-03 4.753060440D-07 $-1.371809730 \mathrm{D}-11$ 1.605374600D-16 $2.488354660 \mathrm{D}+06-6.695524190 \mathrm{D}+02$ IH2 Ref-Elm. TPIS 1978 v1 pt1 p107 pt2 p31.

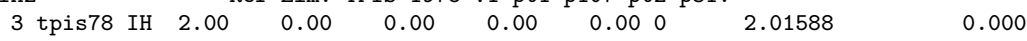

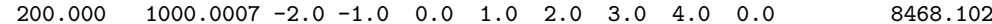

$4.078322810 \mathrm{D}+04-8.009185450 \mathrm{D}+02 \quad 8.214701670 \mathrm{D}+00-1.269714360 \mathrm{D}-02 \quad 1.753604930 \mathrm{D}-05$

$\begin{array}{rrr}-1.202860160 \mathrm{D}-08 & 3.368093160 \mathrm{D}-12 & 2.682484380 \mathrm{D}+03-3.043788660 \mathrm{D}+01\end{array}$

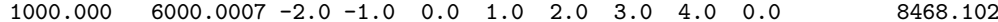

$5.608123380 \mathrm{D}+05-8.371491340 \mathrm{D}+02 \quad 2.975363040 \mathrm{D}+00 \quad 1.252249930 \mathrm{D}-03-3.740718420 \mathrm{D}-07$

$5.936628250 \mathrm{D}-11-3.606995730 \mathrm{D}-15 \quad 5.339815850 \mathrm{D}+03-2.202764050 \mathrm{D}+00$

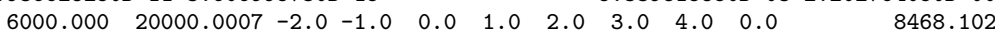

4.966716130D+08-3.147448120D+05 7.983887500D+01-8.414504190D-03 4.753060440D-07 -1.371809730D-11 1.605374600D-16 2.488354660D+06-6.695524190D+02 H2O CODATA 1989. JRNBS 1987 v92 p35. TRC tuv-25 10/88.

$\begin{array}{lllllllll}2 \mathrm{~g} 8 / 89 \mathrm{H} & 2.000 & 1.00 & 0.00 & 0.00 & 0.00 & 0 & 18.01528 & -241826.000\end{array}$

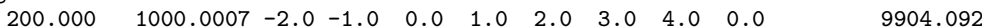

-3.947960830D+04 5.755731020D+02 9.317826530D-01 7.222712860D-03-7.342557370D-06

$\begin{array}{lll}4.955043490 \mathrm{D}-09-1.336933246 \mathrm{D}-12 & -3.303974310 \mathrm{D}+04 & 1.724205775 \mathrm{D}+01\end{array}$

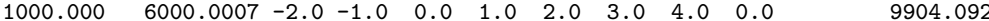
$\begin{array}{llll}1.034972096 \mathrm{D}+06-2.412698562 \mathrm{D}+03 & 4.646110780 \mathrm{D}+00 & 2.291998307 \mathrm{D}-03-6.836830480 \mathrm{D}-07\end{array}$ $9.426468930 \mathrm{D}-11-4.822380530 \mathrm{D}-15 \quad-1.384286509 \mathrm{D}+04-7.978148510 \mathrm{D}+00$ H202 Cons:TPIS 1978 v1 pt1 p121.Hf:TPIS 1989 v1 pt1 p127.

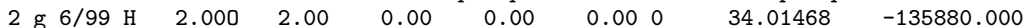

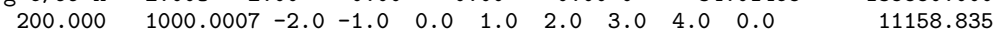
$\begin{array}{llll}-9.279533580 \mathrm{D}+04 & 1.564748385 \mathrm{D}+03-5.976460140 \mathrm{D}+00 & 3.270744520 \mathrm{D}-02-3.932193260 \mathrm{D}-05\end{array}$ $2.509255235 \mathrm{D}-08-6.465045290 \mathrm{D}-12 \quad-2.494004728 \mathrm{D}+04 \quad 5.877174180 \mathrm{D}+01$

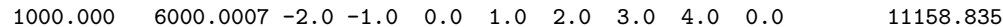
$1.489428027 \mathrm{D}+06-5.170821780 \mathrm{D}+03 \quad 1.128204970 \mathrm{D}+01-8.042397790 \mathrm{D}-05-1.818383769 \mathrm{D}-08$ 6.947265590D-12-4.827831900D-16 1.418251038D+04-4.650855660D+01 $\mathrm{N}$

Hf:CODATA1989, Spec:NSRDS-NBS 3 sec5 1975.

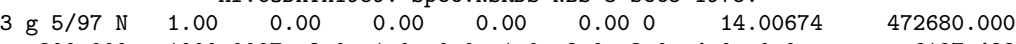

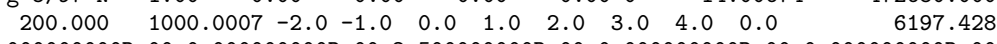
$\begin{array}{lllllll}0.000000000 D+00 & 0.000000000 D+00 & 2.500000000 D+00 & 0.000000000 D+00 & 0.000000000 D+00\end{array}$ $0.000000000 \mathrm{D}+00 \quad 0.000000000 \mathrm{D}+00 \quad 5.610463780 \mathrm{D}+04 \quad 4.193909320 \mathrm{D}+00$

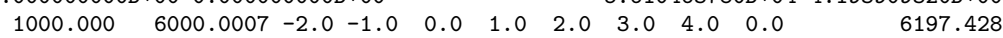
$\begin{array}{lllll}8.876501380 \mathrm{D}+04-1.071231500 \mathrm{D}+02 & 2.362188287 \mathrm{D}+00 & 2.916720081 \mathrm{D}-04-1.729515100 \mathrm{D}-07\end{array}$ 4.012657880D-11-2.677227571D-15 5.697351330D+04 4.865235790D+00

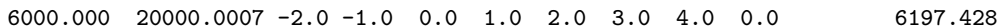
$5.475181050 \mathrm{D}+08-3.107574980 \mathrm{D}+05 \quad 6.916782740 \mathrm{D}+01-6.847988130 \mathrm{D}-03 \quad 3.827572400 \mathrm{D}-07$

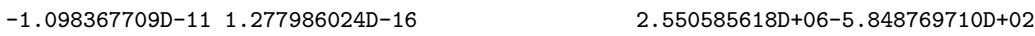
NH3 TPIS $1989 \mathrm{p} 219$. JRNBS 1968 v72A p207 for low T.

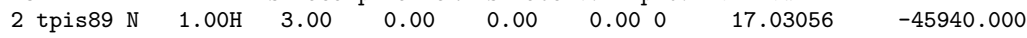

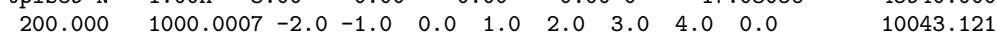

$\begin{array}{lll}-7.681226150 D+04 & 1.270951578 D+03-3.893229130 D+00 & 2.145988418 D-02-2.183766703 D-05\end{array}$

$1.317385706 \mathrm{D}-08-3.332322060 \mathrm{D}-12 \quad-1.264886413 \mathrm{D}+04 \quad 4.366014940 \mathrm{D}+01$

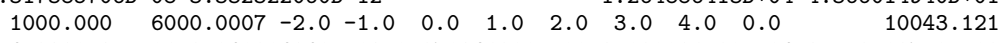
$2.452389535 \mathrm{D}+06-8.040894240 \mathrm{D}+03 \quad 1.271346201 \mathrm{D}+01-3.980186580 \mathrm{D}-04 \quad 3.552502750 \mathrm{D}-08$ $2.530923570 \mathrm{D}-12-3.322700530 \mathrm{D}-16 \quad 4.386191960 \mathrm{D}+04-6.462330250 \mathrm{D}+01$ NO TPIS 1978,1989 v1 pt1 p326 pt2 p203.

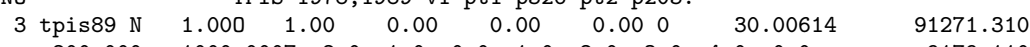

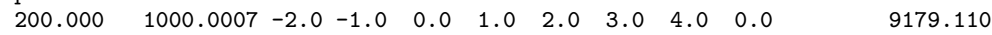

$\begin{array}{llll}-1.143916503 \mathrm{D}+04 & 1.536467592 \mathrm{D}+02 & 3.431468730 \mathrm{D}+00-2.668592368 \mathrm{D}-03 & 8.481399120 \mathrm{D}-06\end{array}$

$\begin{array}{llll}-7.685111050 \mathrm{D}-09 & 2.386797655 \mathrm{D}-12 & 9.098214410 \mathrm{D}+03 & 6.728727490 \mathrm{D}+00\end{array}$

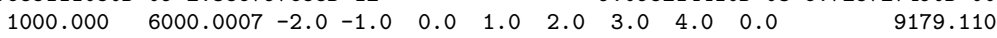

$2.239018716 \mathrm{D}+05-1.289651623 \mathrm{D}+03 \quad 5.433936030 \mathrm{D}+00-3.656034900 \mathrm{D}-04 \quad 9.880966450 \mathrm{D}-08$

$-1.416076856 \mathrm{D}-119.380184620 \mathrm{D}-16 \quad 1.750317656 \mathrm{D}+04-8.501667090 \mathrm{D}+00$

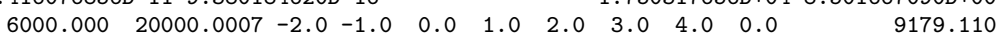
$-9.575303540 \mathrm{D}+08 \quad 5.912434480 \mathrm{D}+05-1.384566826 \mathrm{D}+02 \quad 1.694339403 \mathrm{D}-02-1.007351096 \mathrm{D}-06$ $2.912584076 \mathrm{D}-11-3.295109350 \mathrm{D}-16 \quad-4.677501240 \mathrm{D}+06 \quad 1.242081218 \mathrm{D}+03$ NO2 Hf0,Cons: TPIS 1989 v1 pt1 p332 pt2 p207.

$\begin{array}{lllllllll}2 \text { g } 4 / 99 \mathrm{~N} & 1.000 & 2.00 & 0.00 & 0.00 & 0.00 & 0 & 46.00554 & 34193.019\end{array}$

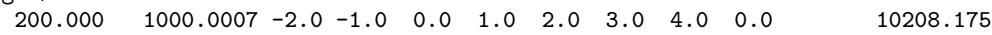

$-5.642038780 \mathrm{D}+04 \quad 9.633085720 \mathrm{D}+02-2.434510974 \mathrm{D}+00 \quad 1.927760886 \mathrm{D}-02-1.874559328 \mathrm{D}-05$

$9.145497730 \mathrm{D}-09-1.777647635 \mathrm{D}-12 \quad-1.547925037 \mathrm{D}+03 \quad 4.067851340 \mathrm{D}+01$

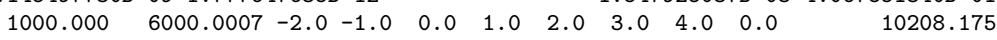

$7.213001570 \mathrm{D}+05-3.832615200 \mathrm{D}+03 \quad 1.113963285 \mathrm{D}+01-2.238062246 \mathrm{D}-03 \quad 6.547723430 \mathrm{D}-07$ $-7.611335900 \mathrm{D}-113.328361050 \mathrm{D}-15$ $2.502497403 \mathrm{D}+04-4.305129910 \mathrm{D}+01$ N03 JPCRD 1998 Mono. 9 p1607.

$\begin{array}{lrrrrrrrr}2 j 12 / 64 \mathrm{~N} & 1.000 & 3.00 & 0.00 & 0.00 & 0.00 & 0 & 62.00494 & 71128.000\end{array}$

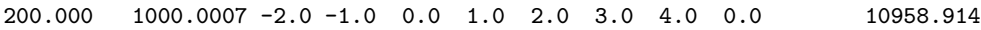


$3.405398410 \mathrm{D}+042.266670652 \mathrm{D}+02-3.793081630 \mathrm{D}+00 \quad 4.170732700 \mathrm{D}-02-5.709913270 \mathrm{D}-05$ 3.834158110D-08-1.021969284D-11 $\quad 7.088112200 \mathrm{D}+03 \quad 4.273091810 \mathrm{D}+01$

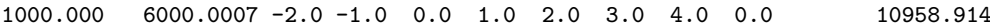
$-3.943872710 \mathrm{D}+05-8.244263530 \mathrm{D}+02 \quad 1.061325843 \mathrm{D}+01-2.448749816 \mathrm{D}-04 \quad 5.406060320 \mathrm{D}-08$ $\begin{array}{lll}-6.195466750 \mathrm{D}-12 & 2.870000149 \mathrm{D}-16 & 8.982011730 \mathrm{D}+03-3.444666500 \mathrm{D}+01\end{array}$ N2 Ref-Elm. TPIS 1978 v1 pt1 p280 pt2 p207.

$\begin{array}{llllllllr}3 \text { tpis78 N } & 2.00 & 0.00 & 0.00 & 0.00 & 0.00 & 0 & 28.01348 & 0.000\end{array}$

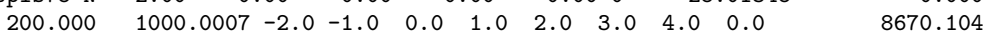
$2.210371497 \mathrm{D}+04-3.818461820 \mathrm{D}+02 \quad 6.082738360 \mathrm{D}+00-8.530914410 \mathrm{D}-03 \quad 1.384646189 \mathrm{D}-05$ $\begin{array}{lll}-9.625793620 \mathrm{D}-09 & 2.519705809 \mathrm{D}-12 & 7.108460860 \mathrm{D}+02-1.076003316 \mathrm{D}+01\end{array}$

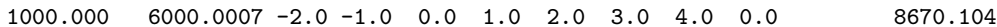
$5.877124060 \mathrm{D}+05-2.239249073 \mathrm{D}+03 \quad 6.066949220 \mathrm{D}+00-6.139685500 \mathrm{D}-04 \quad 1.491806679 \mathrm{D}-07$ $-1.923105485 \mathrm{D}-111.061954386 \mathrm{D}-15 \quad 1.283210415 \mathrm{D}+04-1.586639599 \mathrm{D}+01$

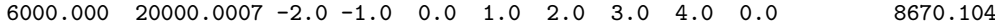
$8.310139160 \mathrm{D}+08-6.420733540 \mathrm{D}+05 \quad 2.020264635 \mathrm{D}+02-3.065092046 \mathrm{D}-02 \quad 2.486903333 \mathrm{D}-06$ $\begin{array}{lll}-9.705954110 \mathrm{D}-11 & 1.437538881 \mathrm{D}-15 & 4.938707040 \mathrm{D}+06-1.672099736 \mathrm{D}+03\end{array}$ $0 \quad$ DO(02):CJP v32 $1954 \mathrm{p} 110$. Spec:NSRDS-NBS 3 sect 1976.

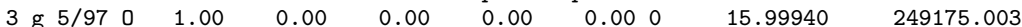

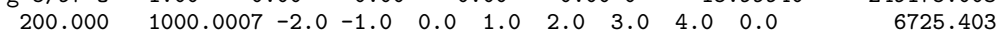

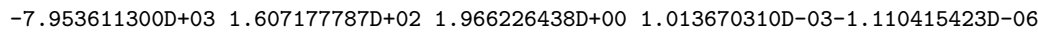
6.517507500D-10-1.584779251D-13 $2.840362437 \mathrm{D}+04 \quad 8.404241820 \mathrm{D}+00$

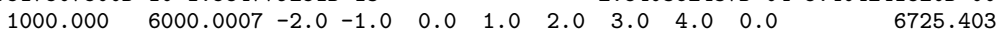
$2.619020262 \mathrm{D}+05-7.298722030 \mathrm{D}+02 \quad 3.317177270 \mathrm{D}+00-4.281334360 \mathrm{D}-04 \quad 1.036104594 \mathrm{D}-07$ $\begin{array}{lll}-9.438304330 \mathrm{D}-12 & 2.725038297 \mathrm{D}-16 & 3.392428060 \mathrm{D}+04-6.679585350 \mathrm{D}-01\end{array}$

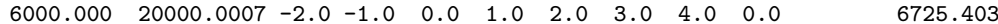
$1.779004264 \mathrm{D}+08-1.082328257 \mathrm{D}+05 \quad 2.810778365 \mathrm{D}+01-2.975232262 \mathrm{D}-03 \quad 1.854997534 \mathrm{D}-07$ $\begin{array}{lll}-5.796231540 \mathrm{D}-12 & 7.191720164 \mathrm{D}-17 & 8.890942630 \mathrm{D}+05-2.181728151 \mathrm{D}+02\end{array}$ IO DO(02):CJP v32 $1954 \mathrm{p} 110$. Spec:NSRDS-NBS 3 sect 1976.

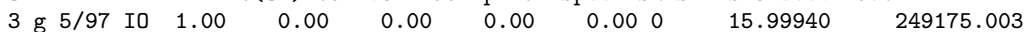

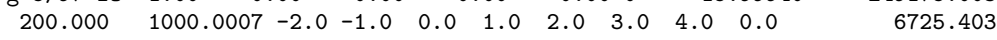
$\begin{array}{llll}-7.953611300 \mathrm{D}+03 & 1.607177787 \mathrm{D}+02 & 1.966226438 \mathrm{D}+00 & 1.013670310 \mathrm{D}-03-1.110415423 \mathrm{D}-06\end{array}$ $6.517507500 \mathrm{D}-10-1.584779251 \mathrm{D}-13 \quad 2.840362437 \mathrm{D}+04 \quad 8.404241820 \mathrm{D}+00$

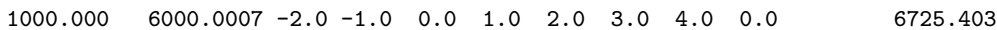
$2.619020262 \mathrm{D}+05-7.298722030 \mathrm{D}+02 \quad 3.317177270 \mathrm{D}+00-4.281334360 \mathrm{D}-04 \quad 1.036104594 \mathrm{D}-07$ $\begin{array}{lll}-9.438304330 \mathrm{D}-12 & 2.725038297 \mathrm{D}-16 & 3.392428060 \mathrm{D}+04-6.679585350 \mathrm{D}-01\end{array}$

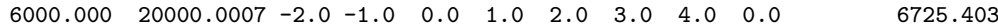
$1.779004264 \mathrm{D}+08-1.082328257 \mathrm{D}+05 \quad 2.810778365 \mathrm{D}+01-2.975232262 \mathrm{D}-03 \quad 1.854997534 \mathrm{D}-07$ $\begin{array}{lll}-5.796231540 \mathrm{D}-12 & 7.191720164 \mathrm{D}-17 & 8.890942630 \mathrm{D}+05-2.181728151 \mathrm{D}+02\end{array}$ $\mathrm{OH}$

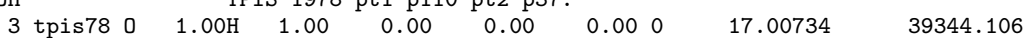

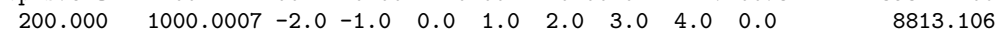
$\begin{array}{llll}-1.998858990 \mathrm{D}+03 & 9.300136160 \mathrm{D}+01 & 3.050854229 \mathrm{D}+00 & 1.529529288 \mathrm{D}-03-3.157890998 \mathrm{D}-06\end{array}$

3.315446180D-09-1.138762683D-12 3.239683480D+03 4.674110790D+00

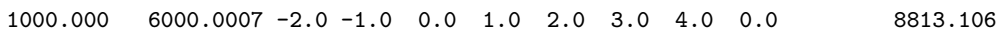
$1.017393379 \mathrm{D}+06-2.509957276 \mathrm{D}+03 \quad 5.116547860 \mathrm{D}+00 \quad 1.305299930 \mathrm{D}-04-8.284322260 \mathrm{D}-08$ $2.006475941 \mathrm{D}-11-1.556993656 \mathrm{D}-15 \quad 2.044487130 \mathrm{D}+04-1.101282337 \mathrm{D}+01$

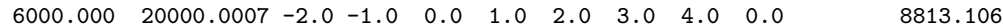
$2.847234193 \mathrm{D}+08-1.859532612 \mathrm{D}+05 \quad 5.008240900 \mathrm{D}+01-5.142374980 \mathrm{D}-03 \quad 2.875536589 \mathrm{D}-07$ $\begin{array}{lll}-8.228817960 \mathrm{D}-12 & 9.567229020 \mathrm{D}-17 & 1.468642377 \mathrm{D}+06-4.023555580 \mathrm{D}+02\end{array}$ 02

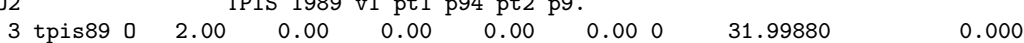

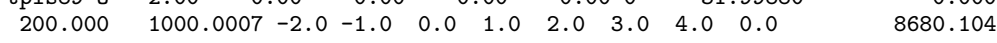
$\begin{array}{llll}-3.425563420 \mathrm{D}+04 & 4.847000970 \mathrm{D}+02 & 1.119010961 \mathrm{D}+00 & 4.293889240 \mathrm{D}-03-6.836300520 \mathrm{D}-07\end{array}$ $\begin{array}{llll}-2.023372700 \mathrm{D}-09 & 1.039040018 \mathrm{D}-12 & -3.391454870 \mathrm{D}+03 & 1.849699470 \mathrm{D}+01\end{array}$

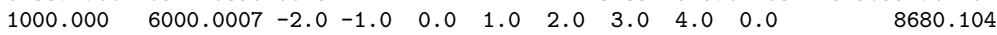
$\begin{array}{llll}-1.037939022 \mathrm{D}+06 & 2.344830282 \mathrm{D}+03 & 1.819732036 \mathrm{D}+00 & 1.267847582 \mathrm{D}-03-2.188067988 \mathrm{D}-07\end{array}$ $2.053719572 \mathrm{D}-11-8.193467050 \mathrm{D}-16 \quad-1.689010929 \mathrm{D}+04 \quad 1.738716506 \mathrm{D}+01$

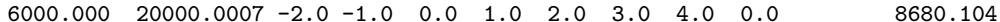
$4.975294300 \mathrm{D}+08-2.866106874 \mathrm{D}+05 \quad 6.690352250 \mathrm{D}+01-6.169959020 \mathrm{D}-03 \quad 3.016396027 \mathrm{D}-07$ $\begin{array}{lll}-7.421416600 \mathrm{D}-12 & 7.278175770 \mathrm{D}-17 & 2.293554027 \mathrm{D}+06-5.530621610 \mathrm{D}+02\end{array}$ I02 TPIS 1989 v1 pt1 p94 pt2 p9.

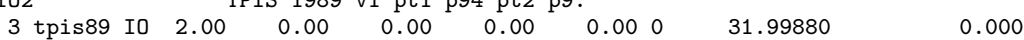

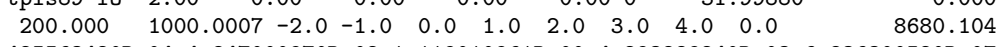
$-3.425563420 \mathrm{D}+04 \quad 4.847000970 \mathrm{D}+02 \quad 1.119010961 \mathrm{D}+00 \quad 4.293889240 \mathrm{D}-03-6.836300520 \mathrm{D}-07$ $\begin{array}{llll}-2.023372700 \mathrm{D}-09 & 1.039040018 \mathrm{D}-12 & -3.391454870 \mathrm{D}+03 & 1.849699470 \mathrm{D}+01\end{array}$

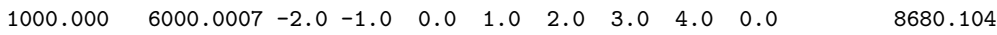
$\begin{array}{lllll}-1.037939022 \mathrm{D}+06 & 2.344830282 \mathrm{D}+03 & 1.819732036 \mathrm{D}+00 & 1.267847582 \mathrm{D}-03-2.188067988 \mathrm{D}-07\end{array}$ $2.053719572 \mathrm{D}-11-8.193467050 \mathrm{D}-16 \quad-1.689010929 \mathrm{D}+04 \quad 1.738716506 \mathrm{D}+01$

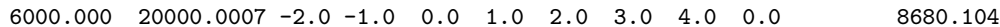
$4.975294300 \mathrm{D}+08-2.866106874 \mathrm{D}+05 \quad 6.690352250 \mathrm{D}+01-6.169959020 \mathrm{D}-03 \quad 3.016396027 \mathrm{D}-07$ $-7.421416600 \mathrm{D}-127.278175770 \mathrm{D}-17 \quad 2.293554027 \mathrm{D}+06-5.530621610 \mathrm{D}+02$ H2O (s) ICE. SANFORD GORDON, NASA TP-1906, 1982.

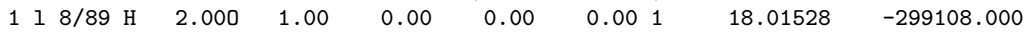

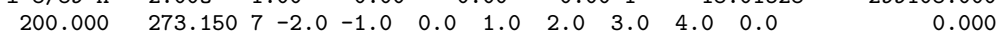
$\begin{array}{llllll}-3.89736288 \mathrm{D}+05 & 2.41502204 \mathrm{D}+03 & 6.09419068 \mathrm{D}+01 & -8.47031137 \mathrm{D}-01 & 4.47768117 \mathrm{D}-03\end{array}$ $\begin{array}{llllll}-1.06521862 \mathrm{D}-05 & 9.77189851 \mathrm{D}-09 & 0.00000000 \mathrm{D}+00 & -5.39150616 \mathrm{D}+04 & -2.07596481 \mathrm{D}+02\end{array}$ $\mathrm{H} 2 \mathrm{O}$ (L) COX ET.AL., CODATA KEY VALUES FOR THERMODYNAMICS. 1989.

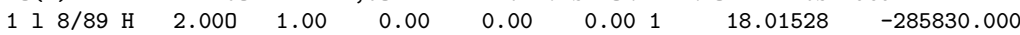

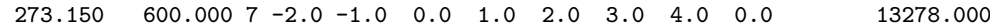
$8.72123781 \mathrm{D}+07-1.39087511 \mathrm{D}+06 \quad 9.15729532 \mathrm{D}+03-3.17596351 \mathrm{D}+01 \quad 6.13885076 \mathrm{D}-02$ $\begin{array}{lllll}-6.26798865 \mathrm{D}-05 & 2.64536349 \mathrm{D}-08 & 0.00000000 \mathrm{D}+00 & 6.43316523 \mathrm{D}+06 & -4.90985319 \mathrm{D}+04\end{array}$ END PRODUCTS

Air Mole\%:N2 78.084,02 20.9476,Ar .9365,C02 .0319.NASA TP1906 1982

20 of 21 


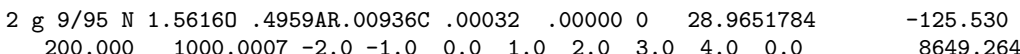

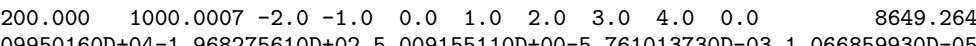
$\begin{array}{rrr}1.009950160 \mathrm{D}+04-1.968275610 \mathrm{D}+02 & 5.009155110 \mathrm{D}+00-5.761013730 \mathrm{D}-03 & 1.066859930 \mathrm{D}-05 \\ -7.940297970 \mathrm{D}-09 & 2.185231910 \mathrm{D}-12 & -1.767967310 \mathrm{D}+02-3.921500990 \mathrm{D}+00\end{array}$

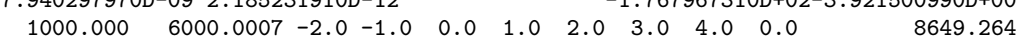
$2.415214430 \mathrm{D}+05-1.257874600 \mathrm{D}+03 \quad 5.144558670 \mathrm{D}+00-2.138541790 \mathrm{D}-04 \quad 7.065227840 \mathrm{D}-08$ $-1.071483490 \mathrm{D}-116.577800150 \mathrm{D}-16 \quad 6.462263190 \mathrm{D}+03-8.147408670 \mathrm{D}+00$ Inert Air Mole\%:N2 78.084,I02 20.9476,Ar.9365,C02 .0319.NASA TP1906 1982

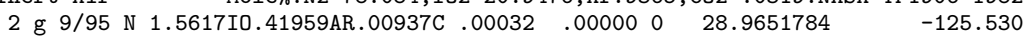

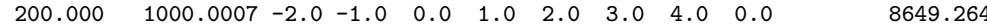

$1.009950160 \mathrm{D}+04-1.968275610 \mathrm{D}+02 \quad 5.009155110 \mathrm{D}+00-5.761013730 \mathrm{D}-03 \quad 1.066859930 \mathrm{D}-05$

$\begin{array}{lll}-7.940297970 \mathrm{D}-09 & 2.185231910 \mathrm{D}-12 & -1.767967310 \mathrm{D}+02-3.921500990 \mathrm{D}+00\end{array}$

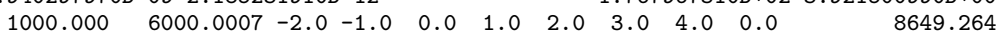

$2.415214430 \mathrm{D}+05-1.257874600 \mathrm{D}+03 \quad 5.144558670 \mathrm{D}+00-2.138541790 \mathrm{D}-04 \quad 7.065227840 \mathrm{D}-08$

-1.071483490D-11 6.577800150D-16 6.462263190D+03-8.147408670D+00

$\mathrm{H} 2$ (L) Hydrogen. JANAF Prop.Ser.D,3/66.

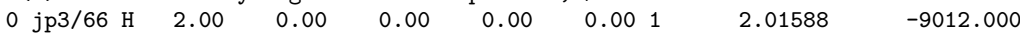
20.270

JP_7 $\begin{array}{llllllllll}20.270 & 0.0000 & 0.0 & 0.0 & 0.0 & 0.0 & 0.0 & 0.0 & 0.0 & 0.0\end{array}$

$$
0.000
$$

$\begin{array}{cccccc}\text { NASA C. Snyder }-9 / 17 / 2001 . & \text { Hcomb } & =18875 . \text { BTU/\# } & \\ \text { OH 2.0044 } 0.00 & 0.00 & 0.001 & 14.03102 & -4745.000\end{array}$

\begin{tabular}{lllllllllr} 
g $5 / 95 \mathrm{C}$ & $1.00 \mathrm{H}$ & 2.0044 & 0.00 & 0.00 & 0.00 & 1 & 14.03102 & -4745.000 \\
\hline
\end{tabular}

$\mathrm{JP}-7$

NASA C. Snyder $-9 / 17 / 2001$. Hcomb $=18875$. BTU $/ \#$

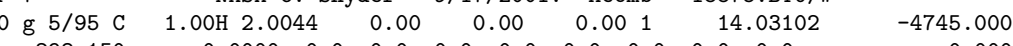
$\begin{array}{llllllllllr}298.150 & 0.0000 & 0.0 & 0.0 & 0.0 & 0.0 & 0.0 & 0.0 & 0.0 & 0.0 & 0.000\end{array}$

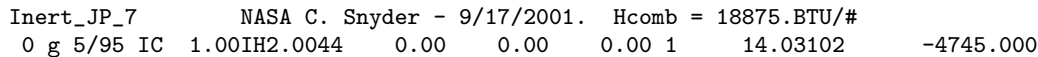

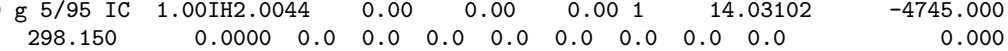

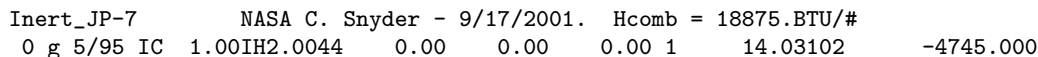

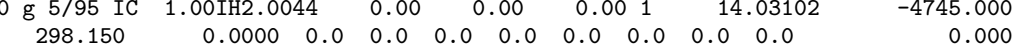

02(L) Oxygen. NASA RP-1311 PtII 1996.

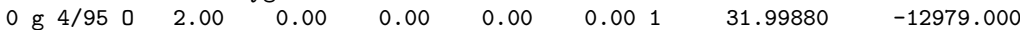
$\begin{array}{lllllllllll}90.170 & 0.0000 & 0.0 & 0.0 & 0.0 & 0.0 & 0.0 & 0.0 & 0.0 & 0.0 & 0.000\end{array}$

Inert_02(L) Oxygen. NASA RP-1311 PtII 1996.

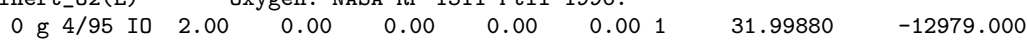
$\begin{array}{lllllllllll}90.170 & 0.0000 & 0.0 & 0.0 & 0.0 & 0.0 & 0.0 & 0.0 & 0.0 & 0.0 & 0.000\end{array}$

JP_4 Or RP-1(L). NASA RP-1311, Part II, 1996. Hcomb $=18640 . \mathrm{BTU} / \#$

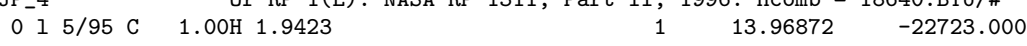
298.15

$\mathrm{JP}-4$ $\begin{array}{lllll}1.00 \mathrm{H} & 1.9423 & 1 & 13.96872 & -22723.000\end{array}$

Or ASTMA1(L). NASA RP-1311, Part II, 1996. Hcomb $=18600 . \mathrm{BTU} / \#$

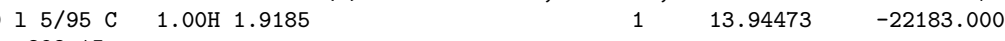
298.15

Jet-A(L) NASA TM-101475,1988. Hcomb=18500 BTU/\#:NASA CR-72951,1971.

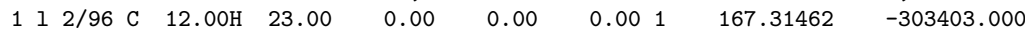

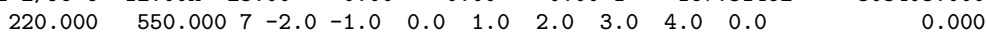
$-4.218467340 \mathrm{D}+05-5.576234840 \mathrm{D}+03 \quad 1.522094335 \mathrm{D}+02-8.610096140 \mathrm{D}-013.071640926 \mathrm{D}-03$ -4.702766120D-06 2.743009309D-09 0.000000000D+00-3.238535350D+04-6.780954740D+02 Jet-A (g) NASA TM-101475,1988. NASA CR-72951,1971.

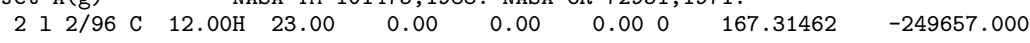

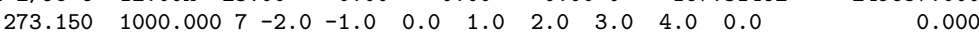
$-6.068699280 \mathrm{D}+05 \quad 8.328264220 \mathrm{D}+03-4.312323550 \mathrm{D}+012.572391032 \mathrm{D}-01-2.629316827 \mathrm{D}-04$ $\begin{array}{llll}1.644989491 \mathrm{D}-07-4.645336690 \mathrm{D}-11 & 0.000000000 \mathrm{D}+00-7.606965040 \mathrm{D}+04 \quad 2.794307229 \mathrm{D}+02\end{array}$

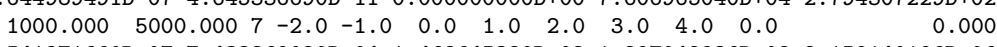

$1.541871660 \mathrm{D}+07-7.433869020 \mathrm{D}+04 \quad 1.468645380 \mathrm{D}+02-1.297042936 \mathrm{D}-02 \quad 2.159140196 \mathrm{D}-06$ $\begin{array}{llll}-1.887183642 \mathrm{D}-10 & 6.604559540 \mathrm{D}-15 & 0.000000000 \mathrm{D}+00 & 3.996323340 \mathrm{D}+05-9.266674660 \mathrm{D}+02\end{array}$ END REACTANTS 